\title{
Simultaneous formation of solar system giant planets
}

\author{
O. M. Guilera ${ }^{1,2, \star}$, A. Fortier ${ }^{3}$, A. Brunini ${ }^{1,2, \star \star}$, and O. G. Benvenuto ${ }^{1,2, \star \star \star}$ \\ 1 Facultad de Ciencias Astronómicas y Geofísicas, Universidad Nacional de La Plata, Paseo del Bosque \\ s/n (B1900FWA) La Plata, Argentina \\ e-mail: [oguilera; abrunini ; obenvenu]@fcaglp.unlp.edu .ar \\ 2 Instituto de Astrofísica de La Plata, IALP, CCT-CONICET-UNLP, Argentina \\ 3 Physikalisches Institut, University of Bern, Sidlerstrasse 5, 3012 Bern, Switzerland \\ e-mail: andrea.fortier@space.unibe.ch
}

Received 10 September 2010 / Accepted 10 May 2011

\begin{abstract}
Context. In the last few years, the so-called "Nice model" has become increasingly significant for studying the formation and evolution of the solar system. According to this model, the initial orbital configuration of the giant planets was much more compact than the one we observe today.

Aims. We study the formation of the giant planets in connection with several parameters that describe the protoplanetary disk. We aim to establish which conditions enable their simultaneous formation in line with the initial configuration proposed by the Nice model. We focus on the conditions that lead to the simultaneous formation of two massive cores, corresponding to Jupiter and Saturn, which are able to reach the cross-over mass (where the mass of the envelope of the giant planet equals the mass of the core, and gaseous runway starts), while two other cores that correspond to Uranus and Neptune have to be able to grow to their current masses.

Methods. We compute the in situ planetary formation, employing the numerical code introduced in our previous work for different density profiles of the protoplanetary disk. Planetesimal migration is taken into account and planetesimals are considered to follow a size distribution between $r_{\mathrm{p}}^{\min }$ (free parameter) and $r_{\mathrm{p}}^{\max }=100 \mathrm{~km}$. The core's growth is computed according to the oligarchic growth regime.

Results. The simultaneous formation of the giant planets was successfully completed for several initial conditions of the disk. We find that for protoplanetary disks characterized by a power law $\left(\Sigma \propto r^{-p}\right)$, flat surface density profiles $(p \leq 1.5)$ favor the simultaneous formation. However, for steep slopes ( $p \sim 2$, as previously proposed by other authors) the simultaneous formation of the solar system giant planets is unlikely.

Conclusions. The simultaneous formation of the giant planets - in the context of the Nice model - is favored by flat surface density profiles. The formation time-scale agrees with the estimates of disk lifetimes if a significant mass of the solids accreted by the planets is contained in planetesimals with radii $<1 \mathrm{~km}$.
\end{abstract}

Key words. planets and satellites: formation - planet-disk interactions - methods: numerical

\section{Introduction}

The initial configuration of the Nice model (Tsiganis et al. 2005; Gomes et al. 2005; Morbidelli et al. 2005) represents the orbital configuration of the outer solar system after the gas of the primordial nebula dissipated. The model assumes that the giant planets were initially in nearly circular and coplanar orbits. This is compatible with the work of Thommes et al. (2008), who find planetary system analogs to our solar system provided that the gas giants do not undergo significant migration during their formation and remain in nearly circular orbits. The Nice model proposes a compact initial configuration for the location of the giant planets; more precisely, the giant planet system is assumed to be in the range of $\sim 5.5 \mathrm{AU}$ to $\sim 14 \mathrm{AU}$. The gas giants, Jupiter and Saturn, are supposed to be close to their mutual 2:1 mean motion resonance (MMR); Jupiter located at $\sim 5.5 \mathrm{AU}$ and Saturn

^ Fellow of the Consejo Nacional de Investigaciones Científicas y Técnicas (CONICET), Argentina.

$\star \star$ Member of the Carrera del Investigador Científico, CONICET, Argentina.

$\star \star \star$ Member of the Carrera del Investigador Científico, Comisión de Investigaciones Científicas de la Provincia de Buenos Aires, Argentina. between 8-8.5 AU. This is an important condition, required to avoid the migration of both planets during the gas disk lifetime (Masset \& Snellgrove 2001; Morbidelli \& Crida 2007). The ice giants are assumed to be located at $\sim 11$ and $\sim 14$ AU.

Another important key of the Nice model is the existence of a planetesimal disk beyond the orbit of the giant planets. The inner edge of the disk is proposed to be located at $\sim 16 \mathrm{AU}$ and the outer edge is fixed at $\sim 30 \mathrm{AU}$; the total mass of the planetesimal disk being $\sim 35-40 M_{\oplus}$. The planetesimals conforming this disk gravitationally interact with the giant planets and cause the inward migration of Jupiter and the outward migration of Saturn to their current positions. In this process, Jupiter and Saturn cross their mutual 2:1 MMR, leading the system to undergo a phase of dynamical instability. During this phase, Uranus and Neptune chaotically migrate outward. According to the numerical simulations of Tsiganis et al. (2005), there is a 50\% probability that the two icy planets switch places in the process.

The success of the Nice model relies on the fact that it is able to explain quantitatively the final orbits, eccentricities, and inclinations of the giant planets (Tsiganis et al. 2005); the chaotic capture of Jupiter's Trojan asteroids (Morbidelli et al. 2005); the origin of the Late Heavy Bombardment (Gomes et al. 2005); the 
formation of the Kuiper belt (Levison et al. 2008) and the secular architecture of the outer solar system (Morbidelli et al. 2009b). Remarkably, the Nice model does not say anything about how its own initial conditions could be achieved.

More recently, Morbidelli \& Crida (2007) - following the work of Masset \& Snellgrove (2001) - showed that for certain parameters of the gas disk, Jupiter and Saturn did not migrate once both planets were locked in 2:3 MMR. Morbidelli \& Crida (2007) showed that this kind of no-migrating (or slowly migrating) evolution is possible if the mass ratio of the two planets is similar to the mass ratio of Jupiter and Saturn. Then, Morbidelli et al. (2007) extended this study to the four giant planets of the solar system. They locked Jupiter and Saturn in 2:3 MMR and found that the inner ice giant could be trapped in the 2:3 or 3:4 MMR with Saturn. Then, the outer ice giant could be trapped in the $3: 4,4: 5$, or 5:6 MMR with the inner ice giant. They showed that the resonant structure is preserved until the gas is completely dispersed. Then, only two configurations were dynamically stable for several hundred million years. Finally, they showed that the existence of a planetesimal disk beyond the outer ice giant led to a dynamical instability, and the essential ingredients of the Nice model are preserved. Batygin \& Brown (2010) extended this work and found more possible multi-resonant initial conditions, several of which evolved in the presence of a planetesimal disk beyond the ice giants, preserving the essential ingredients of the Nice model. While these initial conditions are not exactly the same as those proposed by the Nice model, all of them propose a compact orbital configuration for the giant planets of the solar system in a similar way as the Nice model. However, all these studies assumed that the giant planets were already formed, and did not address the question of how they formed.

Desch (2007) was the first to investigate this. Adopting the in situ formation and perfect accretion for the giant planets, he recalculated the "minimum-mass solar nebula" considering the initial more compact configuration of the giant planets together with the remnant planetesimal disk, as proposed by the Nice model. He then derived a much steeper minimum mass solar nebula surface density profile $\left(\Sigma \propto a^{-2.168}\right.$, where $a$ is the distance to the Sun) than the classical one $\left(\Sigma \propto a^{-3 / 2}\right.$, Weidenschilling 1977; Hayashi 1981). He also estimated the growth time for the cores of the four giant planets. Assuming the oligarchic growth regime for the solid embryos, relative velocities in equilibrium, and a single size population of planetesimals with radii of $100 \mathrm{~m}$, he obtained growth times of about $0.5 \mathrm{Myr}$ for Jupiter, 1.5-2.0 My for Saturn, 5.5-6.0 Myr for Neptune, and 9.5-10.5 Myr for Uranus. However, he did not consider the presence of the also growing gas envelope of the planets.

In a more realistic way, adopting the initial configuration for the location of the giant planets as proposed by the Nice model (Jupiter at 5.5 AU, Saturn at 8.3 AU, Neptune at $11 \mathrm{AU}$, and Uranus at $14 \mathrm{AU}$ ), and employing the surface density profile derived by Desch (2007), Benvenuto et al. (2009) calculated the in situ, isolated formation of the giant planets of the solar system. They used the code already introduced in previous works (Benvenuto \& Brunini 2005; Fortier et al. 2007, 2009) but incorporated a size distribution for the planetesimals radii and considered values between $30 \mathrm{~m}-100 \mathrm{~km}$ (nine species geometrically evenly spaced), with a mass distribution $n(m) \propto m^{-\alpha}$, with $\alpha=2.5$. The choice of $r_{\mathrm{p}}^{\mathrm{min}}=30 \mathrm{~m}$ as the minimum radius of the size distribution of planetesimals relied on a simple estimate for the planetesimal radius, for which the migration timescale of smaller planetesimals should be shorter than the protoplanet's planetesimal accretion time-scale, which means that the accretion of planetesimals with radii $<30 \mathrm{~m}$ should be not as efficient. Imposing a value of $11 \mathrm{~g} \mathrm{~cm}^{-2}$ for the solids' surface density at the location of Jupiter, Benvenuto et al. calculated the formation time for Jupiter (0.44 Myr), Saturn (1.4 Myr), Neptune (2.5 Myr), and Uranus (4.75 Myr). Furthermore, they found that the final core masses of the four planets agreed very well with the present theoretical estimate. However, some important simplifications were assumed in that work. It is well known that planet formation, disk evolution and planetesimal migration occur on the same time scale. Consequently, a closer approach to the formation of the giant planets should include an approximation of the other two processes. In Benvenuto et al. (2009) and also in Desch (2007) the migration of planetesimals owing to the gas drag of the disk was not taken into account. As Thommes et al. (2003), Chambers (2006), and Brunini \& Benvenuto (2008) showed, this effect has a strong influence on the accretion time, especially for small planetesimals. Therefore, planetesimal migration should be incorporated in a more realistic model. On the other hand, the evolution of the gaseous component of the disk should be taken into account, because the gas density is an input parameter when determining the accretion rates and the planetesimal migration velocities. Finally, the planetesimals' relative velocities were considered to be in equilibrium. But, it is not clear that an equilibrium condition is ever achieved, specially for small planetesimals (Chambers 2006; Brunini \& Benvenuto 2008). Instead, relative velocities out of equilibrium should be adopted because the size distribution of the planetesimals extends to meter-size objects.

These conditions were incorporated in a recent model developed by Guilera et al. (2010). They calculated the simultaneous formation of Jupiter and Saturn at their current locations immersed in a protoplanetary disk that evolves with time. They found that the simultaneous formation of Jupiter and Saturn could be very different compared to the isolated formation of each planet. For the classical solar system surface density profile $\left(\Sigma \propto a^{-3 / 2}\right)$, they found that the formation of Jupiter inhibits the growth of Saturn. On the other hand, for a disk with surface density profile $\Sigma \propto a^{-1 / 2}$, Saturn turned out to form faster than Jupiter. Moreover, the quick formation of Saturn produced an inner solid density wave that significantly reduced the formation time of Jupiter. Finally, for a disk with a surface density profile $\Sigma \propto a^{-1}$, simultaneous and isolated formation of both planets resulted in very similar outcomes.

The aim of this work is to study some of the parameters that characterize the protoplanetary disk to determine the conditions that could favor/hinder the formation of the solar system giant planets, in line with the initial configuration proposed by the Nice model. The formation of the planets is calculated using the in situ approximation. Additionally, the formation of the four planets are computed simultaneously, which means that the formation of one planet can affect the formation of another one because it perturbs the planetesimal disk. We will therefore focus our analysis on the first stage of the planetary formation, i.e., before the gaseous runaway starts. We aim to gain insight into the conditions of the disk (especially the surface density profiles and the planetesimal size distribution) that lead to the formation of the cores of Jupiter and Saturn on a timescale that enables the planets to reach the gaseous runaway. Moreover, Uranus and Neptune should also be able to grow to their current mass. For this purpose, we consider a population of planetesimals with non homogeneous radii that follow a power law distribution, where the radii go from $r_{\mathrm{p}}^{\mathrm{min}}$ to $100 \mathrm{~km}$ (the maximum value of the distribution is fixed for all cases). The value of the minimum radius is to be determined as follows: we first calculate the isolated 
formation of the four planets for different values of $r_{\mathrm{p}}^{\min }$ and then determine the optimum values they can reach (by "optimum values" we mean those values of $r_{\mathrm{p}}^{\min }$ that the four planets have in common that minimize their time of formation). With these values we then explore the simultaneous formation. This procedure is employed to analyze different profiles for the surface density of the protoplanetary disk. Finally, we discuss the scenario that best matches the basic observational constraints for the formation of the giant planets (the disk dissipation time scale and the estimated mass of the core) with the conditions of the Nice model.

The remainder of this paper is organized as follows: in Sect. 2 we give a brief description of the model we developed in a previous work (Guilera et al. 2010). In Sect. 3 we calculate the formation of the giant planets of the solar system for a steep surface density profile similar to the one derived by Desch (2007). Then, we present the results for flatter surface density profiles in Sect. 4. Finally, in Sect. 5 we discuss our results and present the concluding remarks.

\section{A brief description of the model}

In a previous work (Guilera et al. 2010), we developed a numerical code to compute the in situ, simultaneous formation of several giant planets immersed in a protoplanetary disk that evolves with time. In the present paper, we use this code to study the first stage of the formation of the four giant planets of the solar system. We here briefly recall the main points.

The protoplanetary disk was characterized by gaseous and solid (a planetesimal disk) components. We considered a classic power-law to describe the surface density, $\Sigma \propto a^{-p}$. In our model, the disk extends from 0.4 to $30 \mathrm{AU}$. We fixed the outer edge of the disk at $30 \mathrm{AU}$ to be consistent with the outer edge of the planetesimal disk used by the Nice model. For the planetesimal disk, we considered a population of non-equal sized bodies. Planetesimals were assumed to follow a size distribution whose radii were between a minimum value, $r_{\mathrm{p}}^{\mathrm{min}}$, and $100 \mathrm{~km}$, with steps selected so that the quotient of masses of consecutive sizes is a factor of two. For example, the continuous planetesimal size distribution between $1 \mathrm{~km}$ and $100 \mathrm{~km}$ was characterized by 21 different sizes of planetesimals, while the continuous planetesimal size distribution between $10 \mathrm{~m}$ and $100 \mathrm{~km}$ was characterized by 41 different sizes of planetesimals. Kokubo \& Ida (2000), employing $N$-body simulations, and more recently, Ormel et al. (2010), employing statistical simulations, showed that in the oligarchic growth regime planetesimals follow a mass distribution $\mathrm{d} n / \mathrm{d} m \propto m^{-p}$, with $p \sim 2.5$. We adopted this distribution for this work. Note that most of the mass is contained in small planetesimals.

As we mentioned above, we considered the protoplanetary disk to evolve. In terms of the planetesimal disk, we considered planetesimal migration owing to nebular gas drag. We adopted the migration velocities given by the prescription of Adachi et al. (1976),

$$
\frac{\mathrm{d} a}{\mathrm{~d} t}=-\frac{2 a}{T_{\text {fric }}}\left(\eta^{2}+\frac{5}{8} e^{2}+\frac{1}{2} i^{2}\right)^{1 / 2}\left[\eta+\left(\frac{5}{16}+\frac{\alpha}{4}\right) e^{2}+\frac{1}{4} i^{2}\right],
$$

where

$$
T_{\text {fric }}=\frac{8 \rho_{\mathrm{p}} r_{\mathrm{p}}}{3 C_{\mathrm{D}} \rho_{\mathrm{g}} v_{\mathrm{k}}} .
$$

Here $\rho_{\mathrm{p}}$ is the bulk density of planetesimals $\left(1.5 \mathrm{~g} \mathrm{~cm}^{-2}\right), r_{\mathrm{p}}$ is the radius of the planetesimal, $C_{\mathrm{D}} \sim 1, \eta=\left(v_{\mathrm{k}}-v_{\mathrm{gas}}\right) / v_{\mathrm{k}}$, and $\alpha$ is the exponent of the power-law density of the gas in the disk mid plane $\left(\rho_{\mathrm{g}} \propto a^{-\alpha}\right)$. Then, the evolution of the planetesimals disk obeys a continuity equation,

$\frac{\partial \Sigma_{\mathrm{s}}}{\partial t}-\frac{1}{a} \frac{\partial}{\partial a}\left(a \frac{\mathrm{d} a}{\mathrm{~d} t} \Sigma_{\mathrm{s}}\right)=F(a)$,

where $\Sigma_{\mathrm{s}}$ is the surface density of solids and $F(a)$ describes the sinks of disk material (accretion by the forming planets).

Adopting a temperature profile for an optically thin disk $(T \propto$ $\left.a^{-1 / 2}\right)$, the density distribution of gas on the mid-plane of the disk is given by $\rho_{\mathrm{g}} \propto a^{-p-5 / 4}$, and we assumed for simplicity that the gaseous component dissipates out following an exponential decay of its density with a characteristic time-scale of $6 \mathrm{Myr}$ (Haisch et al. 2001),

$\rho_{\mathrm{g}}(a, t)=\rho_{\mathrm{g}}(a, 0) \mathrm{e}^{-t / 6 \mathrm{Myr}}$.

We adopted the oligarchic regime for the growth of the core in accordance with our previous work (Guilera et al. 2010). Adopting the particle in a box approximation (Inaba et al. 2001) for the accretion of solids

$\frac{\mathrm{d} M_{\mathrm{C}}}{\mathrm{d} t}=\frac{2 \pi \Sigma_{\mathrm{s}}\left(a_{\mathrm{P}}\right) R_{\mathrm{H}}^{2}}{P} P_{\text {coll }}$,

and integrating over the planetesimals size distribution and the feeding zone, the solid accretion rate is given by

$$
\begin{aligned}
\frac{\mathrm{d} M_{\mathrm{C}}}{\mathrm{d} t} & =\int_{\mathrm{PSD}} \mathrm{d} m \int_{\mathrm{FZ}} 2 \pi \psi\left(a, R_{\mathrm{H}}, a_{P}\right) \\
& \times \frac{2 \pi \Sigma_{\mathrm{s}}(a, m) R_{\mathrm{H}}^{2}}{P} P_{\mathrm{coll}}(a, m) a \mathrm{~d} a,
\end{aligned}
$$

where $M_{\mathrm{C}}$ is the mass of the core, $R_{\mathrm{H}}$ is the Hill radius, $P$ is the orbital period, $a_{\mathrm{P}}$ is the semi-major axis of the planet, and $\psi$ is a normalization function (see Brunini \& Benvenuto 2008). $P_{\text {coll }}$ is the collision probability between the planetesimals and the planet, which is a function of the planet's core radius, the Hill radius of the planet, and the relative velocity of planetesimals $P_{\text {coll }}=P_{\text {coll }}\left(R_{\mathrm{C}}, R_{\mathrm{H}}, v_{\text {rel }}\right)$ (for more details, see Guilera et al. 2010).

We also took into account the enhancement of the planet's capture cross-section owing to the presence of the planet's envelope. Inaba \& Ikoma (2003) found an approximate solution to the equations of motion, which allows for a rapid estimation of the radius of the planetesimal captured, $r_{\mathrm{p}}$, as function of the the planet's enhanced radius $\tilde{R}_{\mathrm{C}}$

$r_{\mathrm{p}}=\frac{3 \rho\left(\tilde{R}_{\mathrm{C}}\right) \tilde{R}_{\mathrm{C}}}{2 \rho_{\mathrm{p}}}\left(\frac{v_{\infty}^{2}+2 G M_{\mathrm{P}}\left(\tilde{R}_{\mathrm{C}}\right) / \tilde{R}_{\mathrm{C}}}{v_{\infty}^{2}+2 G M_{\mathrm{P}}\left(\tilde{R}_{\mathrm{C}}\right) / R_{\mathrm{H}}}\right)$,

where $v_{\infty}$ is the relative velocity of the planet and planetesimal when the two are far apart, while $M_{\mathrm{P}}\left(\tilde{R}_{\mathrm{C}}\right)$ and $\rho\left(\tilde{R}_{\mathrm{C}}\right)$ are the total mass of the planet and the density of the planet's envelope contained within $\tilde{R}_{\mathrm{C}}$, respectively. Inaba \& Ikoma (2003) propose replacing $\tilde{R}_{\mathrm{C}}$ for $R_{\mathrm{C}}$ in the expressions of collision probability, so $P_{\text {coll }}=P_{\text {coll }}\left(\tilde{R}_{\mathrm{C}}, R_{\mathrm{H}}, v_{\text {rel }}\right)$.

Planetesimal relative velocities out of equilibrium were prescribed. The relative velocity, $v_{\text {rel }}$, between a planetesimal and the protoplanet may be described by

$v_{\mathrm{rel}}=\sqrt{\frac{5}{8} e^{2}+\frac{1}{2} i^{2}} v_{\mathrm{k}}$ 
where $v_{\mathrm{k}}$ is the Keplerian velocity. We considered that the relative velocity is governed by two factors, the gravitational stirring caused by the protoplanets and the gas drag damping. We adopted the prescriptions developed by Ohtsuki et al. (2002) for the gravitational stirring and the prescriptions developed by Adachi et al. (1976) for the gas drag damping (see Guilera et al. 2010, for a more detailed explanation).

Finally, the equations governing the evolution of the gaseous envelope are those classic of stellar evolution theory

$$
\begin{aligned}
\frac{\partial r}{\partial m_{r}}=\frac{1}{4 \pi r^{2} \rho} & \text { equation of definition of mass } \\
\frac{\partial P}{\partial m_{r}}=-\frac{G m_{r}}{4 \pi r^{4}} & \text { equation of hydrostatic equilibrium } \\
\frac{\partial L_{r}}{\partial m_{r}}=\epsilon_{\mathrm{pl}}-T \frac{\partial S}{\partial t} & \text { equation of energetic balance } \\
\frac{\partial T}{\partial m_{r}}=-\frac{G m_{r} T}{4 \pi r^{4} P} \nabla & \text { equation of energy transport, }
\end{aligned}
$$

where $\rho$ is the density of the envelope, $G$ is the universal gravitational constant, $\epsilon_{\mathrm{pl}}$ is the energy release rate due to the accretion of planetesimals, $\mathrm{S}$ is the entropy per unit mass, and $\nabla \equiv \frac{\mathrm{d} \ln T}{\mathrm{~d} \ln P}$ is the dimensionless temperature gradient, which depends on the type of energy transport.

These equations were solved coupled self-consistently to the planetesimal's accretion rate (Eq. (6)), employing a standard finite difference (Henyey) method and a detailed constitutive physics as described in Fortier et al. (2007, 2009), and Guilera et al. (2010).

In the following sections we present our results for the simultaneous, in situ formation of the four giant planets of the solar system. Note that planets were assumed to be in circular orbits around the Sun with fixed orbital radii consistent with the Nice model. Accordingly, the migration of the planets is not addressed in the present study.

Thommes et al. (2008) showed that solar system analogs develop if gas giants do form but undergo modest migration and eccentricity growth, even for massive protoplanetary disks. Miguel et al. (2010a,b) did a statistical study of the formation of planetary systems and also found that solar system analogs are favored in massive disks where there is no large accumulation of solids in the inner region of the disk, and only if type I migration is strongly reduced. Both works showed that toward low disk masses, planet formation is too slow to produce gas giants during the disk lifetime.

Several authors have argued that type I migration should be much slower than previously thought, otherwise planets get too close to or are even engulfed by the star (see, for instance, Alibert et al. 2005, for a detailed calculation of the formation of Jupiter and Saturn including migration). Tanaka et al. (2002) derived the migration rate for vertically isothermal disks. However, it was recently found that migration rates, and even the direction of migration, could be very different when vertically radiative or adiabatic transfer for the protoplanetary disk is adopted (Kley et al. 2009; Paardekooper et al. 2010). In light of recent results that suggest type I migration might not be as important as previously thought in more realistic disks (i.e. not the idealized case proposed by Tanaka et al.), we find our hypothesis of in situ formation to be a good approximation to a more complex and realistic model, at least at early stages of the formation process.

The exclusion of type II migration is more problematic given that this is likely to be an important effect in many systems, especially because the Nice model and its sequels call for an early migration and multi-resonant capture of the giant planets. However, calculating type II migration rates is a complicated and subtle procedure in multi-planet systems and is beyond the scope of this paper. It is important therefore to caution here that our results should be considered in the context of the in situ formation, which could be considered as a good approximation during the first stage of the formation of giant planets, i.e. before the gaseous runaway begins.

Our model also excludes the final stages of the gas accretion. Our code calculates the gas accretion rate of the planet selfconsistently using an adapted Henyey-type code of stellar evolution, but always under the assumption that the disk can supply the necessary amount of gas required by the planet. But, after the runaway growth of the envelope sets in, this hypothesis may not be valid any more. However, recent works have shown that gas accretion onto a critical mass core is likely to be rapid. Machida et al. (2010), employing three-dimensional hydrodynamics simulations, found that the gas accretion time-scale of a giant planet is about $10^{5} \mathrm{yr}$ (two orders of magnitude less than the usually estimated time-scale to form the core). This means that the real deciding factor for giant planet formation is the growth of a critical mass core in the first place.

For the above mentioned reasons we think our code is suitable for studying the first stage of planetary formation: the growth of the planets up to their critical masses and how the simultaneous formation differs from the isolated case.

\section{Simultaneous formation of the solar system giant planets for a disk with a steep profile: $\Sigma \propto a^{-2}$}

Adopting the initial configuration proposed by the Nice model, Desch (2007) derived a surface density profile for an analog of the minimum mass solar nebula that can be adjusted with a single power law, $\Sigma \propto a^{-2.168}$. This profile is much steeper than the one derived by Hayashi (1981), where $\Sigma \propto a^{-1.5}$. We therefore started our investigation adopting a similar density profile as that proposed by Desch; in our case, and for the sake of simplicity, we adopted $\Sigma \propto a^{-2}$. Pollack et al. (1996) have used this profile to study the isolated formation of giant planets in solar system, but adopting a faster time-dependent regime for the growth of the core than the one corresponding to the oligarchic growth regime.

Following the work of Benvenuto et al. (2009), we imposed the value of $11 \mathrm{~g} \mathrm{~cm}^{-2}$ for the initial solids' surface density at the location of Jupiter. Then, at $t=0$ when the calculations begin, the density profile throughout the disk is described by

$\Sigma_{\mathrm{s}}=11\left(\frac{a}{5.5 \mathrm{AU}}\right)^{-2} \eta_{\text {ice }} \mathrm{g} \mathrm{cm}^{-2}$

where $\eta_{\text {ice }}$ takes into account the condensation of volatiles beyond the snow line, considered to be located at 2.7 AU,

$\eta_{\text {ice }}= \begin{cases}1 & a>2.7 \mathrm{AU}, \\ \frac{1}{4} & a<2.7 \mathrm{AU} .\end{cases}$

We adopted a value for the gas-to-solid ratio of $Z_{0}^{-1} \simeq 65$, where $Z_{0}=0.0153$ (Lodders et al. 2009) is the initial abundance of heavy elements in the Sun, implying that the initial surface density of gas is given by

$\Sigma_{\mathrm{g}}=719\left(\frac{a}{5.5 \mathrm{AU}}\right)^{-2} \mathrm{~g} \mathrm{~cm}^{-2}$. 
Table 1. Isolated formation of the solar system giant planets' cores for a disk with surface densities of solids and gas $\propto a^{-2}$.

\begin{tabular}{|c|c|c|c|c|c|c|c|c|}
\hline $\begin{array}{l}r_{\mathrm{p}}^{\min } \\
{[m]}\end{array}$ & $\begin{array}{c}\text { Jupiter } \\
M_{\text {over }}^{\text {cross }} \\
{\left[M_{\oplus}\right]} \\
\end{array}$ & $\begin{array}{c}t_{\mathrm{over}}^{\text {cross }} \\
{[\mathrm{Myr}]}\end{array}$ & $\begin{array}{c}\text { Saturn } \\
M_{\text {over }}^{\text {cross }} \\
{\left[M_{\oplus}\right]} \\
\end{array}$ & $\begin{array}{c}t_{\mathrm{over}}^{\text {cross }} \\
{[\mathrm{Myr}]}\end{array}$ & $\begin{array}{c}\text { Neptune } \\
M_{\text {over }}^{\text {cross }} \\
{\left[M_{\oplus}\right]}\end{array}$ & $\begin{array}{c}t_{\mathrm{over}}^{\text {cross }} \\
{[\mathrm{Myr}]}\end{array}$ & $\begin{array}{c}\text { Uranus } \\
M_{\text {overs }}^{\text {cros }} \\
{\left[M_{\oplus}\right]} \\
\end{array}$ & $\begin{array}{c}t_{\text {over }}^{\text {cross }} \\
{[\mathrm{Myr}]}\end{array}$ \\
\hline 10 & - & - & 19.13 & 6.96 & $\begin{array}{c}22.02 \\
(14.62)\end{array}$ & $\begin{array}{c}2.90 \\
(2.31)\end{array}$ & $\begin{array}{c}18.75 \\
(12.59)\end{array}$ & $\begin{array}{c}6.01 \\
(4.98)\end{array}$ \\
\hline 12.5 & - & - & 19.22 & 5.15 & $\begin{array}{l}21.07 \\
(14.57)\end{array}$ & $\begin{array}{c}3.04 \\
(2.43)\end{array}$ & $\begin{array}{l}13.10 \\
(11.24)\end{array}$ & $\begin{array}{c}13.88 \\
(12.39)\end{array}$ \\
\hline 15 & - & - & 19.65 & 3.91 & $\begin{array}{c}19.26 \\
(14.27)\end{array}$ & $\begin{array}{c}3.85 \\
(3.17)\end{array}$ & - & - \\
\hline 20 & - & - & 20.90 & 2.83 & $\begin{array}{c}14.48 \\
(12.78)\end{array}$ & $\begin{array}{c}8.69 \\
(7.82)\end{array}$ & - & - \\
\hline 25 & 12.54 & 14.98 & 20.92 & 2.78 & - & - & - & - \\
\hline 40 & 16.89 & 6.09 & 16.30 & 5.73 & - & - & - & - \\
\hline 50 & 18.34 & 4.38 & 12.87 & 10.43 & - & - & - & - \\
\hline 75 & 18.80 & 3.91 & - & - & - & - & - & - \\
\hline 100 & 17.59 & 4.79 & - & - & - & - & - & - \\
\hline 150 & 15.22 & 7.06 & - & - & - & - & - & - \\
\hline 200 & 13.61 & 9.43 & - & - & - & - & - & - \\
\hline
\end{tabular}

Notes. Here $r_{\mathrm{p}}^{\min }$ stands for the minimum radius of the size distribution of planetesimals. $t_{\mathrm{over}}^{\text {cross }}$ represents the time at which the mass of the envelope of the planet achieves the core's mass of the planet and gaseous runaway starts. $M_{\text {over }}^{\text {cros }}$ is the mass of the core at the cross-over time. Quantities between brackets for Neptune and Uranus correspond to the time and the respective core mass at which Neptune and Uranus achieve its current masses ( 17 and $\sim 14.5$ Earth masses, respectively). When the cross-over time exceeds $\sim 10 \mathrm{Myr}$, no values are given.

Rescaling to $1 \mathrm{AU}$ and spreading the snow line in a region of about $1 \mathrm{AU}$ with a smooth function as proposed by Thommes et al. (2003), the disk was defined as

$$
\begin{aligned}
\Sigma_{\mathrm{s}}(a)= & \left\{83.19+(332.75-83.19)\left[\frac{1}{2} \tanh \left(\frac{a-2.7}{0.5}\right)+\frac{1}{2}\right]\right\} \\
& \times\left(\frac{a}{1 \mathrm{AU}}\right)^{-2} \mathrm{~g} \mathrm{~cm}^{-2} \\
\Sigma_{\mathrm{g}}(a)= & 21750\left(\frac{a}{1 \mathrm{AU}}\right)^{-2} \mathrm{~g} \mathrm{~cm}^{-2} .
\end{aligned}
$$

Regarding the temperature profile, we adopted the same one as in our previous works,

$$
T(a)=280\left(\frac{a}{1 \mathrm{AU}}\right)^{-1 / 2} \mathrm{~K}
$$

We did not consider the evolution in time of the temperature profile, which implies that the temperature of the nebula at a given location is a fixed external boundary condition. The initial mass of the disk was $\sim 0.066 M_{\odot}$, this value being the result of integrating the surface density profile from $a=0.4$ AU to $a=$ $30 \mathrm{AU}$.

With the disk so defined, we first calculated the isolated formation of each of the four giant planets of the solar system. For each planet we ran several simulations only changing the minimum radius of the size distribution of planetesimals, $r_{\mathrm{p}}^{\mathrm{min}}$. The aim of this procedure was to look for an interval in the planetesimal radii where the isolated formation of all the planets occurs in less than 10 Myr. Afterward, and using these results as a guide, we looked for an optimum value of $r_{\mathrm{p}}^{\mathrm{min}}$ to calculate the simultaneous formation of the four planets.

The results for the isolated formation are summarized in Table 1. We found that each planet has an independent interval for $r_{\mathrm{p}}^{\mathrm{min}}$ in which the cross-over time (the time at which the mass

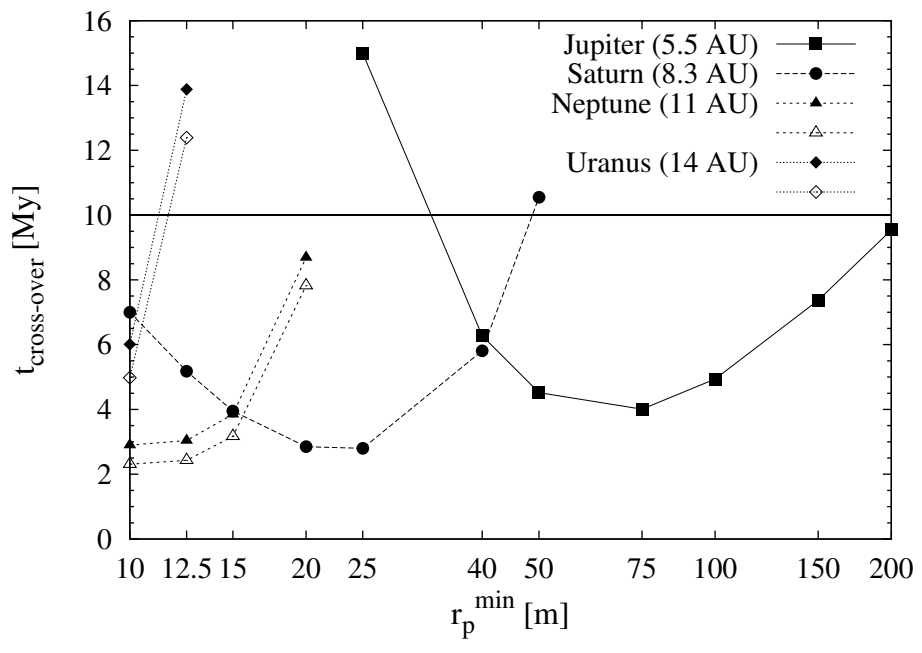

Fig. 1. Cross-over time as function of the minimum radius of the planetesimal size distribution for the isolated formation of each planet. Results correspond to a disk with a surface density profile $\Sigma \propto a^{-2}$. Open triangles (diamonds) correspond to the time at which Neptune (Uranus) achieves its current mass ( $\sim 17$ and $\sim 14.5$ Earth masses, respectively).

of the envelope achieves the core's mass and gaseous runaway starts) is reached in less than $10 \mathrm{Myr}$ (Fig. 1). Jupiter reached its cross-over mass and could be formed in less than $\sim 10 \mathrm{Myr}$ for $r_{\mathrm{p}}^{\mathrm{min}}$ in the range of $\sim 30$ and $200 \mathrm{~m}$. Saturn, on the other hand, reached its cross-over mass in less than $\sim 10$ Myr for $r_{\mathrm{p}}^{\min }$ between 10 and $\sim 50 \mathrm{~m}$. In the case of Neptune, $r_{\mathrm{p}}^{\mathrm{min}}$ had to be between 10 and $20 \mathrm{~m}$. Finally, Uranus could only be formed in agreement with the time restriction for $r_{\mathrm{p}}^{\min } \lesssim 12.5 \mathrm{~m}$.

Note that we did not consider radii smaller than $10 \mathrm{~m}$. This is because in most cases (see below) the time-scale of formation of the outermost planets are significantly lower for these small planetesimals than the time-scale for Jupiter and Saturn. We consider it very unlikely that Uranus and Neptune reached their current masses before Jupiter and Saturn reached their cross-over masses, because in that case they could have continued their growth and become gas giants.

For Desch's steep profile, we found that the shortest crossover time for Jupiter is $\sim 4 \mathrm{Myr}$, for Saturn is $\sim 2.8 \mathrm{Myr}$, for Neptune is $\sim 2.9 \mathrm{Myr}$ and for Uranus is $\sim 6 \mathrm{Myr}$. However, these time-scales did not occur for the same minimum radii of the planetesimals that populate the disk.

On the other hand, we found that for Jupiter and Saturn the cross-over masses agreed well with the current theoretical estimate of their solid content (Fig. 2). Note that we assumed that all the infalling planetesimals reach the core's surface without losing mass on their trajectories throughout the envelope, this meaning that $M_{\mathrm{c}}$ corresponds in reality to the total heavy element's mass in the interior of the planet (core plus solids in the envelope). Theoretical models estimate the core mass of Jupiter between 0-12 $M_{\oplus}$ (Guillot 2005) or 14-18 $M_{\oplus}$ (Militzer et al. 2008), depending on the equation of state employed. The core mass of Saturn is accepted to be in the range of 9-22 $M_{\oplus}$ (Guillot 2005). Models also predict 10-40 $M_{\oplus}$ and 20-30 $M_{\oplus}$ of total heavy elements (envelope + core) in Jupiter and Saturn, respectively (Guillot 2005; Guillot \& Gautier 2009). On the other hand, Uranus and Neptune are mostly rocks and ices. The planetary interior models of Podolak et al. (2000) place upper limits on the $\mathrm{H} / \mathrm{He}$ contents of Uranus and Neptune, which are for Uranus of 4.2 and $3.2 M_{\oplus}$ for Neptune. If only hydrogen and 


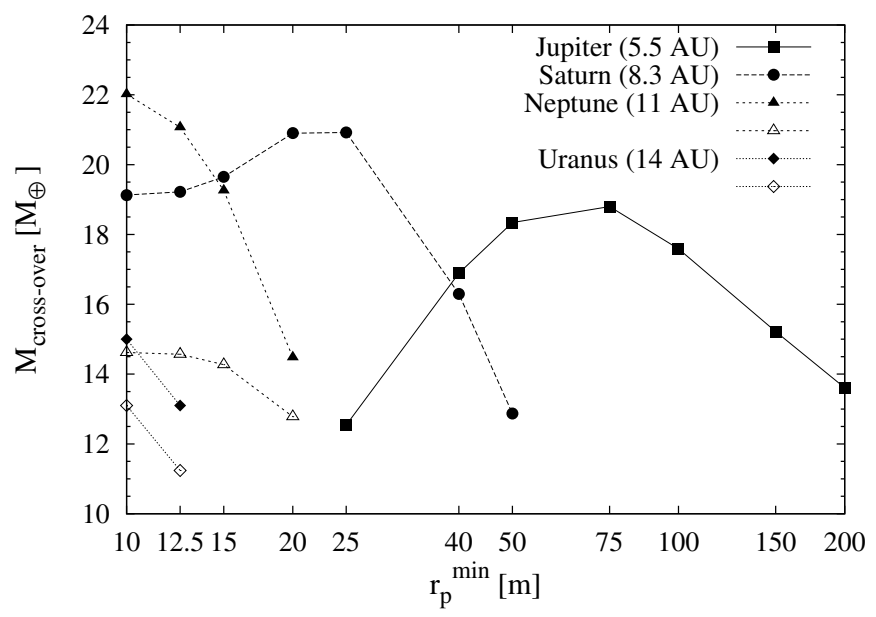

Fig. 2. Core mass at the cross-over time as function of the minimum radius of the planetesimal size distribution for the isolated formation of each planet. Results correspond to a disk with a surface density profile $\Sigma \propto a^{-2}$. Open triangles (diamonds) correspond to a mass of the core at the time in which Neptune (Uranus) achieve their current mass $(\sim 17$ and $\sim 14.5$ Earth masses, respectively).

helium are present in the atmosphere, a lower limit for the gas mass of $0.5 M_{\oplus}$ is obtained for each planet (Guillot 2005). To relate the above mentioned values to our results of the core mass, we will consider below that the total amount of heavy elements contained in each planet corresponds to the mean value of the current estimated boundaries. This means that we are going to compare our results for the mass of the core with $25 \pm 15 M_{\oplus}$ for Jupiter, $25 \pm 5 M_{\oplus}$ for Saturn, 15.15 $\pm 1.35 M_{\oplus}$ for Neptune, and $12.15 \pm 1.85 M_{\oplus}$, for Uranus.

We can see for the formation of Neptune and Uranus (Table 1 and Fig. 2) that if we let them grow without restricting their final masses, their cross-over masses were mostly higher than their current masses. However, the masses of the cores at the time they reached their current masses agree with theoretical estimate.

For Jupiter and Saturn we found an optimum value for $r_{\mathrm{p}}^{\mathrm{min}}$ of around $70 \mathrm{~m}$ for Jupiter, and between 20-25 m for Saturn, for which the accretion was most effective. The existence of an optimum $r_{\mathrm{p}}^{\mathrm{min}}$ for the size distribution of the planetesimal radii is due to the fact that planetesimal's migration velocities are $\propto 1 / r_{\mathrm{p}}$ (Eq. (1)) and that the planetesimal's accretion by the protoplanet is more efficient for small objects. Furthermore, there is a competition between the efficiency of the accretion and the efficiency of the planetesimal migration. Because we considered a planetesimal mass distribution that follows the power law $n(m)=m^{-2.5}$ (where most of the mass of solids lie in the small objects), $r_{\mathrm{p}}^{\mathrm{min}}$ has to be chosen considering the above mentioned compromise between the migration and the accretion rate. If the radii of the smaller planetesimals are very small, most of the mass of solids would pass through the feeding zone of the planet without being accreted. If, on the other hand, they are very large, the accretion time scale would be longer than the protoplanetary disk lifetime.

The intervals for $r_{\mathrm{p}}^{\mathrm{min}}$ of each planet are different becasue both the accretion rate of planetesimals and their migration velocities vary with the distance to the central star. We found no overlap between these intervals for this steep profile so it seems that there is no common size distribution for the planetesimal radii that would allow us to form the four planets simultaneously in less than 10 Myr.
Table 2. Same as Table 1 but for the simultaneous formation.

\begin{tabular}{cccccccccc}
\hline \hline & Jupiter & \multicolumn{3}{c}{ Saturn } & \multicolumn{3}{c}{ Neptune } & \multicolumn{3}{c}{ Uranus } \\
$r_{\mathrm{p}}^{\min }$ & $M_{\text {over }}^{\text {cross }}$ & $t_{\text {over }}^{\text {cross }}$ & $M_{\text {over }}^{\text {cross }}$ & $t_{\text {over }}^{\text {cross }}$ & $M_{\text {over }}^{\text {cros }}$ & $t_{\text {over }}^{\text {cross }}$ & $M_{\text {over }}^{\text {cross }}$ & $t_{\text {over }}^{\text {cross }}$ \\
{$[m]$} & {$\left[M_{\oplus}\right]$} & {$[\mathrm{Myr}]$} & {$\left[M_{\oplus}\right]$} & {$[\mathrm{Myr}]$} & {$\left[M_{\oplus}\right]$} & {$[\mathrm{Myr}]$} & {$\left[M_{\oplus}\right]$} & {$[\mathrm{Myr}]$} \\
\hline 10 & $\sim 0.5$ & $\gg 10$ & 17.73 & 8.73 & 19.81 & 5.16 & 13.61 & 10.26 \\
& & & & & $(14.86)$ & $(4.28)$ & $(11.80)$ & $(8.24)$ \\
50 & 15.47 & 8.89 & 13.42 & 10.44 & $\sim 1$ & $\gg 10$ & $\sim 0.5$ & $\gg 10$ \\
\hline
\end{tabular}

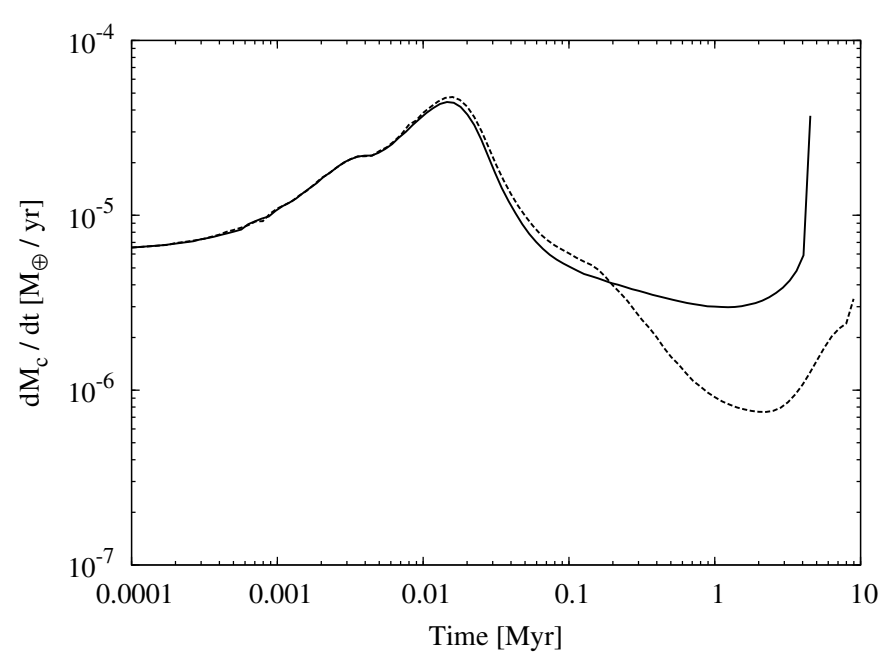

Fig. 3. Time evolution of Jupiter's planetesimal accretion rate for the isolated (solid line) and simultaneous formation (dashed line) for a disk profile $\Sigma \propto a^{-2}$.

However, in spite of the previous results, we checked if there was after all an interval where the simultaneous formation could occur. Taking into account the results for the calculations of isolated formation, we ran some simulations where we considered the simultaneous formation of the four giant planets.

Results are listed in Table 2. We first chose $r_{\mathrm{p}}^{\mathrm{min}}=50 \mathrm{~m}$ for the size distribution of planetesimals. In this case the formation times for Jupiter and Saturn were $\sim 9 \mathrm{Myr}$ and $\sim 10.5 \mathrm{Myr}$, respectively. However, in this time interval the embryos of Neptune and Uranus only reached a core mass of $M_{\mathrm{c}} \sim 1 M_{\oplus}$ and $M_{\mathrm{c}} \sim 0.5 M_{\oplus}$ respectively, both with a negligible envelope (the simulation was stopped at $\sim 11 \mathrm{Myr}$, when Saturn finished its formation). We note that the presence of Saturn delayed the formation time of Jupiter by a factor $\sim 2$ compared to the isolated results. This is shown in Fig. 3. The reduction in the solid accretion rate takes place because planetesimals that were accreted by Jupiter in the isolated case (coming from the outer region of the solar system) were accreted by Saturn first when the formation of both planets was calculated simultaneously. For this steep profile, the presence of Saturn acts as a sink of planetesimals, significantly decreasing the surface density of smaller planetesimals in the neighborhood of Jupiter when we compare it with the isolated formation of Jupiter (see Fig. 4).

When we consider smaller planetesimals $\left(r_{\mathrm{p}}^{\mathrm{min}}=10 \mathrm{~m}\right)$, Neptune turned out to be the first one to achieve its cross-over mass, followed by Saturn and Uranus, while Jupiter was not able to reach its cross-over mass in less than $10 \mathrm{Myr}$ (the simulation was stopped at $\sim 15 \mathrm{Myr}$ and Jupiter's core mass was only $\sim 0.5 M_{\oplus}$ with almost no gas bound). However, in the simultaneous case, the formation of all the planets took longer than in the isolated case. We consider that a case like this is very unlikely because while Jupiter was unable to grow up to its cross-over mass, Neptune reached its cross-over mass before Saturn, which 
O. M. Guilera et al.: Simultaneous formation of solar system giant planets
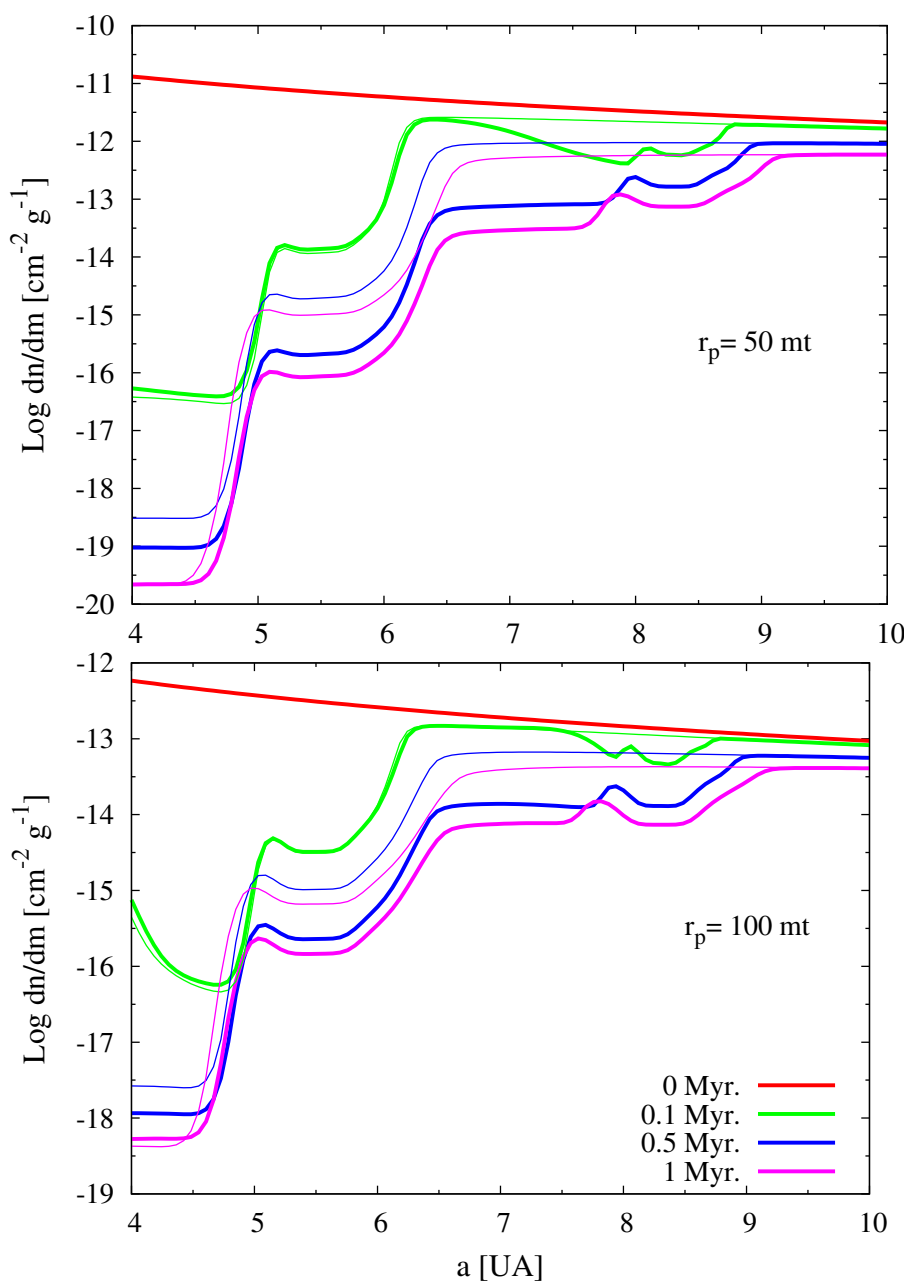

Fig. 4. Time evolution of the surface density of solids for small planetesimals in a disk with power index $p=2$. Thin lines correspond to the isolated formation of Jupiter, thick lines correspond to the simultaneous formation of the giant planets. Evidently, the presence of Saturn significantly reduces the surface density of solids at Jupiter's location by the accretion of small planetesimals. The presence of Saturn delayed the formation time of Jupiter by a factor $\sim 2$ (color figure only available in the electronic version).

means that it could have started the gaseous runaway (and become a gas giant) before Saturn completed its formation. While it is widely accepted that Uranus and Neptune did not reach the gaseous runaway to become gas giants, we remark that we did not halt the accretion onto Uranus and Neptune in our simulations when they reached their current masses and allowed them to grow up to the cross-over mass, because the perturbations that a planet produces on the planetesimal disk and how they affect the formation of the others depends on its mass. This is in line with our working hypothesis of studying the formation of the planets up to their cross-over masses, a process that is well described with our code. In this work we do not attempt to explain the final masses of the planets.

Therefore, it would be necessary for a nebula like the one considered here that $r_{\mathrm{p}}^{\mathrm{min}}$ decreases with the distance from the central star to be able to form the four planets simultaneously. Moreover, an accurate treatment demands that the simultaneous formation should be recalculated with a non homogeneous distribution. However, in principle, there is no physical reason to support such a decrease in the minimum radii of the planetesimals as we move away from the Sun. Indeed, a decrease in
Table 3. Same as Table 1 but for a disk with surface density of solids and gas $\propto a^{-3 / 2}$.

\begin{tabular}{|c|c|c|c|c|c|c|c|c|}
\hline $\begin{array}{l}r_{\mathrm{p}}^{\min } \\
{[m]}\end{array}$ & $\begin{array}{c}\text { Jupiter } \\
M_{\text {over }}^{\text {cross }} \\
{\left[M_{\oplus}\right]} \\
\end{array}$ & $\begin{array}{r}t_{\text {over }}^{\text {cross }} \\
{[\mathrm{Myr}]}\end{array}$ & $\begin{array}{c}\text { Saturn } \\
M_{\text {over }}^{\text {cross }} \\
{\left[M_{\oplus}\right]} \\
\end{array}$ & $\begin{array}{c}t_{\text {over }}^{\text {cross }} \\
{[\mathrm{Myr}]}\end{array}$ & $\begin{array}{c}\text { Neptune } \\
M_{\text {over }}^{\text {cross }} \\
{\left[M_{\oplus}\right]}\end{array}$ & $\begin{array}{c}t_{\mathrm{over}}^{\text {cross }} \\
{[\mathrm{Myr}]}\end{array}$ & $\begin{array}{c}\text { Uranus } \\
M_{\text {over }}^{\text {cros }} \\
{\left[M_{\oplus}\right]} \\
\end{array}$ & $\begin{array}{c}t_{\text {over }}^{\text {cross }} \\
{[\mathrm{Myr}]}\end{array}$ \\
\hline 10 & - & - & 23.49 & 4.14 & $\begin{array}{c}28.28 \\
(15.30)\end{array}$ & $\begin{array}{c}1.10 \\
(0.75)\end{array}$ & $\begin{array}{c}32.20 \\
(13.67)\end{array}$ & $\begin{array}{c}0.61 \\
(0.35)\end{array}$ \\
\hline 20 & 19.88 & 9.85 & 26.93 & 1.33 & $\begin{array}{c}30.33 \\
(15.58)\end{array}$ & $\begin{array}{c}0.87 \\
(0.61)\end{array}$ & $\begin{array}{c}26.15 \\
(13.37)\end{array}$ & $\begin{array}{r}1.91 \\
(1.45)\end{array}$ \\
\hline 35 & 20.92 & 4.58 & 28.38 & 1.22 & $\begin{array}{c}24.02 \\
(15.05)\end{array}$ & $\begin{array}{c}2.52 \\
(2.08)\end{array}$ & $\begin{array}{c}17.57 \\
(12.45)\end{array}$ & $\begin{array}{c}7.62 \\
(6.50)\end{array}$ \\
\hline 40 & 21.65 & 3.76 & 27.53 & 1.44 & $\begin{array}{c}22.33 \\
(14.85)\end{array}$ & $\begin{array}{c}3.28 \\
(2.77)\end{array}$ & $\begin{array}{c}15.19 \\
(11.90)\end{array}$ & $\begin{array}{l}10.92 \\
(9.56)\end{array}$ \\
\hline 80 & 23.67 & 2.61 & 21.08 & 3.90 & $\begin{array}{l}13.11 \\
(12.3)\end{array}$ & $\begin{array}{l}13.20 \\
(12.6)\end{array}$ & $\begin{array}{l}- \\
-\end{array}$ & $\begin{array}{l}- \\
-\end{array}$ \\
\hline 160 & 21.03 & 3.70 & 14.59 & 10.73 & - & - & - & - \\
\hline 400 & 15.98 & 7.81 & - & - & - & $\begin{array}{lll}- & \end{array}$ & - & - \\
\hline
\end{tabular}

the planetesimals' radii would contradict the results found by Chambers (2010), where the planetesimal's size should increase with distance.

\section{Simultaneous formation of the solar system giant planets for shallow disk density profiles}

In the previous section we showed that with a steep profile as the one derived by Desch (2007) for a minimum mass solar nebula compatible with the Nice model, there is no minimum radius for the population of the planetesimals that favors the simultaneous formation of the four giant planets of the solar system. In this section we will analyze other density profiles, all of them power laws for which we vary the value of the index $p$.

\subsection{The case of $\Sigma \propto a^{-3 / 2}$}

Employing the standard surface density profile $\Sigma \propto a^{-3 / 2}$, we repeated the calculations of Sect. 3 and first attended to the isolated formation to look for candidate values for $r_{\mathrm{p}}^{\min }$ and later calculated with these values the simultaneous formation of the four planets.

Imposing the value of $11 \mathrm{~g} \mathrm{~cm}^{2}$ again for the solid surface density at the location of Jupiter, using the same solid/gas ratio, and rescaling to $1 \mathrm{AU}$, the disk is defined by

$$
\begin{aligned}
\Sigma_{\mathrm{s}}(a)= & \left\{35.5+(142-35.5)\left[\frac{1}{2} \tanh \left(\frac{a-2.7}{0.5}\right)+\frac{1}{2}\right]\right\} \\
& \times\left(\frac{a}{1 \mathrm{AU}}\right)^{-3 / 2} \mathrm{~g} \mathrm{~cm}^{-2} \\
\Sigma_{\mathrm{g}}(a)= & 9281\left(\frac{a}{1 \mathrm{AU}}\right)^{-3 / 2} \mathrm{~g} \mathrm{~cm}^{-2} .
\end{aligned}
$$

According to this definition, the initial mass of the disk was $\sim 0.063 M_{\odot}$.

The results obtained for the isolated formation of each planet are summarized in Table 3 . We note that these results are qualitatively similar to those obtained for the steeper profile $\Sigma \propto a^{-2}$. However, in this case there are some values for $r_{\mathrm{p}}^{\min }$ (between $20-40 \mathrm{~m}$ ) that may allow the simultaneous formation of all planets with a common size distribution of planetesimals.

For the isolated formation of each planet, Jupiter reached its cross-over mass in less than $10 \mathrm{Myr}$ for $20 \mathrm{~m} \lesssim r_{\mathrm{p}}^{\mathrm{min}} \lesssim 400 \mathrm{~m}$, Saturn for $10 \mathrm{~m} \lesssim r_{\mathrm{p}}^{\mathrm{min}} \lesssim 160 \mathrm{~m}$, Neptune for $10 \mathrm{~m} \lesssim r_{\mathrm{p}}^{\min } \lesssim$ $80 \mathrm{~m}$, and Uranus for $10 \mathrm{~m} \lesssim r_{\mathrm{p}}^{\mathrm{min}} \lesssim 40 \mathrm{~m}$. Accordingly, there 


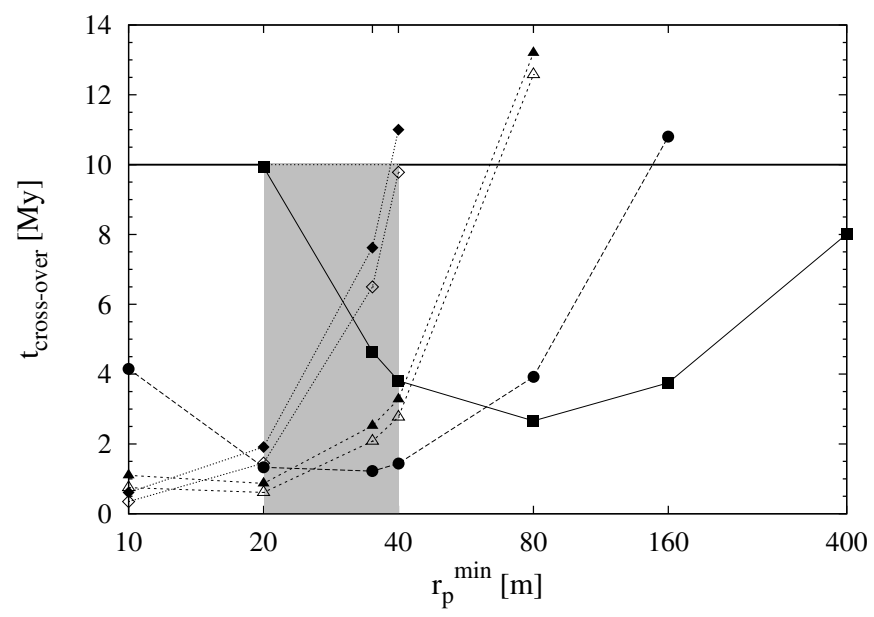

Fig. 5. Same as Fig. 1 but considering a disk with a surface density profile $\Sigma \propto a^{-3 / 2}$. The gray zone corresponds to the interval of values of the minimum radius of the size distribution of planetesimals where the cross-over times corresponding to the four planets are less than $\sim 10$ Myr. Squares, circles, triangles, and diamonds correspond to Jupiter, Saturn, Neptune, and Uranus, respectively.

Table 4. Same as Table 3 but for the simultaneous formation of the four planets.

\begin{tabular}{cccccccccc}
\hline \hline & Jupiter & \multicolumn{3}{c}{ Saturn } & \multicolumn{3}{c}{ Neptune } & \multicolumn{3}{c}{ Uranus } \\
$r_{\mathrm{p}}^{\text {min }}$ & $M_{\text {over }}^{\text {cross }}$ & $t_{\text {over }}^{\text {cross }}$ & $M_{\text {over }}^{\text {coros }}$ & $t_{\text {over }}^{\text {cross }}$ & $M_{\text {over }}^{\text {coros }}$ & $t_{\text {over }}^{\text {cross }}$ & $M_{\text {over }}^{\text {cross }}$ & $t_{\text {over }}^{\text {cross }}$ \\
{$[m]$} & {$\left[M_{\oplus}\right]$} & {$[\mathrm{Myr}]$} & {$\left[M_{\oplus}\right]$} & {$[\mathrm{Myr}]$} & {$\left[M_{\oplus}\right]$} & {$[\mathrm{Myr}]$} & {$\left[M_{\oplus}\right]$} & {$[\mathrm{Myr}]$} \\
\hline 20 & 35.74 & 0.99 & 38.30 & 0.55 & 33.65 & 0.60 & $27.83^{*}$ & $1.6^{*}$ \\
& & & & & $(16.20)$ & $(0.42)$ & $(13.92)$ & $(1.1)$ \\
35 & 31.89 & 2.25 & 29.12 & 1.83 & 23.43 & 3.88 & 16.74 & 8.68 \\
& & & & & $(15.52)$ & $(3.01)$ & $(12.69)$ & $(6.80)$ \\
40 & 30.08 & 2.96 & 27.28 & 2.55 & 20.72 & 5.27 & 14.70 & 12.23 \\
& & & & & $(15.12)$ & $(4.19)$ & $(12.18)$ & $(10.02)$ \\
\hline
\end{tabular}

Notes. ${ }^{(*)}$ For this case, the numerical models for Uranus did not converge (Uranus did not achieve its cross-over mass) and the simulation was halted. At time $t=1.6 \mathrm{Myr}$, Uranus reached a core mass of $27.83 M_{\oplus}$ with a corresponding envelope mass of $8.75 M_{\oplus}$.

should be an optimum radius $r_{\mathrm{p}}^{\min }$ for this protoplanetary nebula where the four planets reach their cross-over masses in less than $10 \mathrm{Myr}$ (Fig. 5), this radius lies in the interval [20, 40] m. Note that for a planetesimal size distribution between $r_{\mathrm{p}}^{\min }=20 \mathrm{~m}$ and $r_{\mathrm{p}}^{\max }=100 \mathrm{~km}$, the first planet to reach its cross-over mass was Neptune, followed by Saturn, Uranus, and Jupiter (see Table 3). However, for a size distribution of planetesimals between $r_{\mathrm{p}}^{\min }=$ $35 \mathrm{~m}$ and $r_{\mathrm{p}}^{\max }=100 \mathrm{~km}$, and between $r_{\mathrm{p}}^{\min }=40 \mathrm{~m}$ and $r_{\mathrm{p}}^{\max }=$ $100 \mathrm{~km}$, the first planet to reach the cross-over mass was Saturn, followed by Neptune, Jupiter and Uranus.

We then calculated the simultaneous formation of the four planets for $r_{\mathrm{p}}^{\mathrm{min}}=20,35$ and $40 \mathrm{~m}$. Results are summarized in Table 4 and Fig. 8. Note that for $r_{\mathrm{p}}^{\min }=20 \mathrm{~m}$ the formation of the system changed drastically. The rapid formation of the outermost planets (especially the rapid formation of Saturn) significantly favored the formation of Jupiter. Similar results were found in our previous work (Guilera et al. 2010) if Saturn was formed before Jupiter. This rapid formation of the outermost planets induced an inner density wave that is responsible for the reduction in the formation time of Jupiter and Saturn. The density wave (see Figs. 6 and 7) is a product of the excitation of the planets over the planetesimals. The gravitational stirring of the
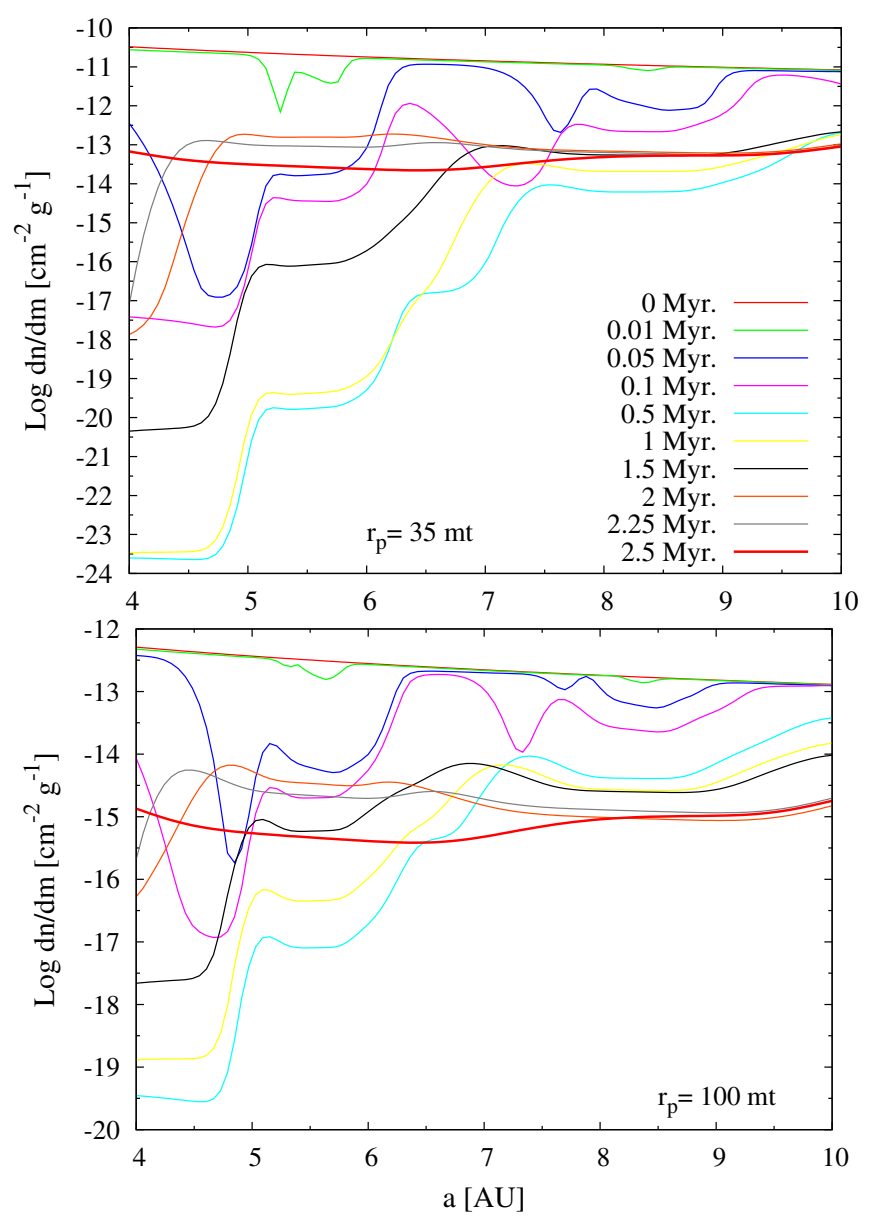

Fig. 6. Time evolution of the surface density of solids for small planetesimals at the Jupiter-Saturn region for a disk with a power index $p=3 / 2$, considering the case of the simultaneous formation and employing a distribution of planetesimals between $r_{\mathrm{p}}^{\min }=35$ and $r_{\mathrm{p}}^{\max }=100 \mathrm{~km}$. After 0.5 Myr. the surface density of solids at Jupiter's neighborhood is significantly augmented by the incoming flow of planetesimals owing to the gravitational perturbations of the outer planets (color figure only available in the electronic version).

planets increases the eccentricity and inclination of the planetesimals and force their inner migration (see Eq. (1)). This enhances the solids's surface density and accelerates the formation of the planet cores. We note that we only considered the simultaneous formation of four embryos. However, oligarchic growth predicts the presence of several embryos separated by $\sim 10$ mutual Hill radii. The presence of several embryos may damp the solid density wave, but may on the other hand also favor the formation of massive cores by their mutual collisions, which may lead to their fusion.

For $r_{\mathrm{p}}^{\mathrm{min}}=35$ and $40 \mathrm{~m}$, the formation of the system was quantitatively different. Although the formation time of Saturn was prolonged by the presence of the other embryos, it could still be considered fast. The rapid formation of Saturn significantly decreased the formation time of Jupiter. As we can see in Figs. 6 and 7, the quick formation of Saturn forced the migration of planetesimals and augmented the surface density of solids in Jupiter's neighborhood. After 0.5 Myr. the surface density of solids in Jupiter's neighborhood was increased for small planetesimals (Fig. 6) because of Saturn. For bigger planetesimals, the shape of the density wave is more evident, and in some cases the value of the surface density of solids become greater than the initial ones (Fig. 7). 
O. M. Guilera et al.: Simultaneous formation of solar system giant planets
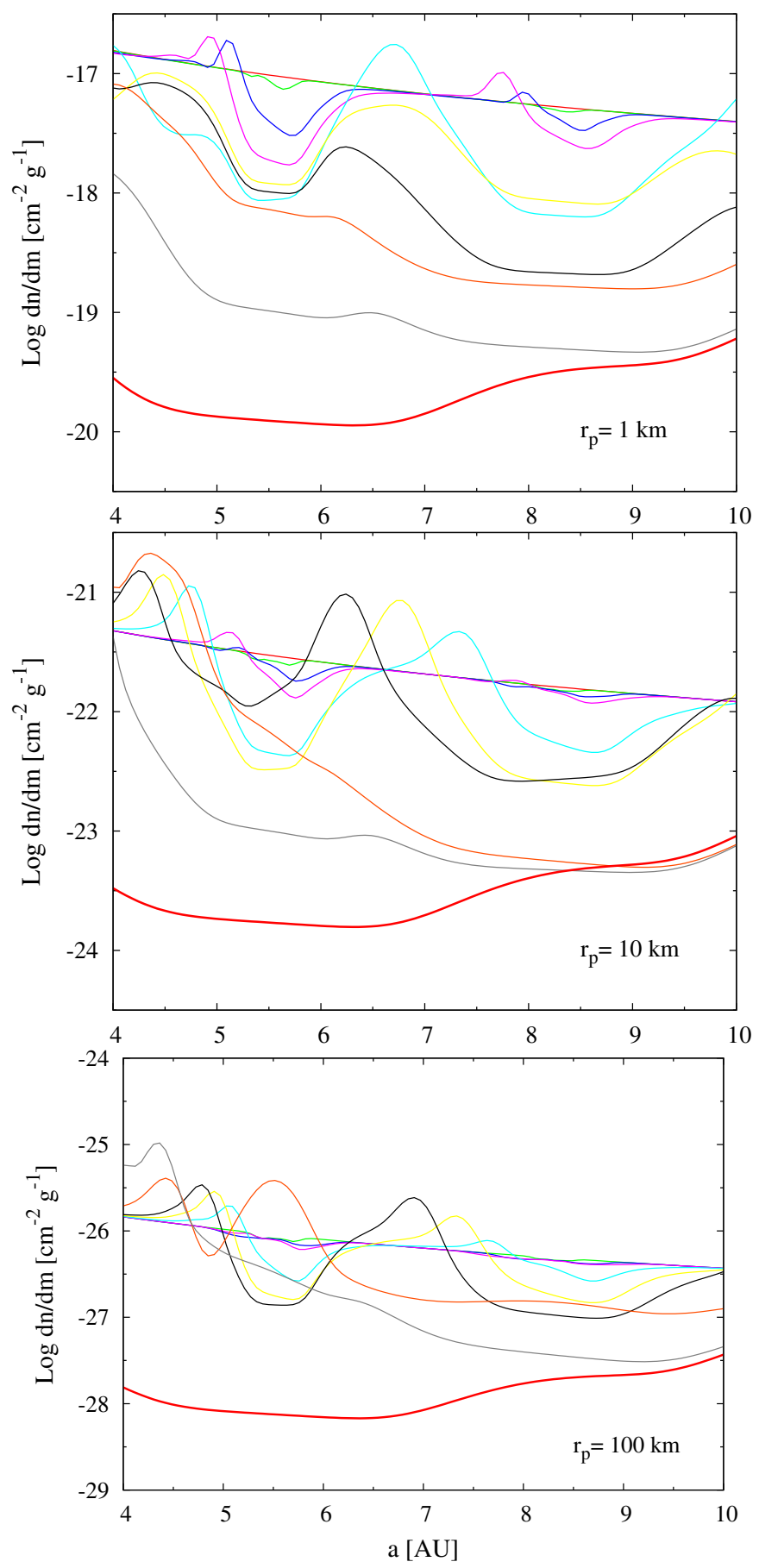

Fig. 7. Same as Fig. 6 but for planetesimals with radii larger than $1 \mathrm{~km}$. In this case the shape of the density wave is well defined. The density wave increases the surface density of solids to values that are even higher than the initial ones (color figure only available in the electronic version).

In these two simulations, the cross-over times and cross-over masses of Jupiter and Saturn agreed well with observations and theoretical estimates of protoplanetary disk lifetimes and current solid contents in the interior of the planets, respectively (Fig. 8). Moreover, the time-scale at which both planets reached the gaseous runaway were very similar. Uranus and Neptune had larger cross-over masses than their current total masses. However, for both planets, the core masses at the time they reached their current masses agreed with theoretical estimates.

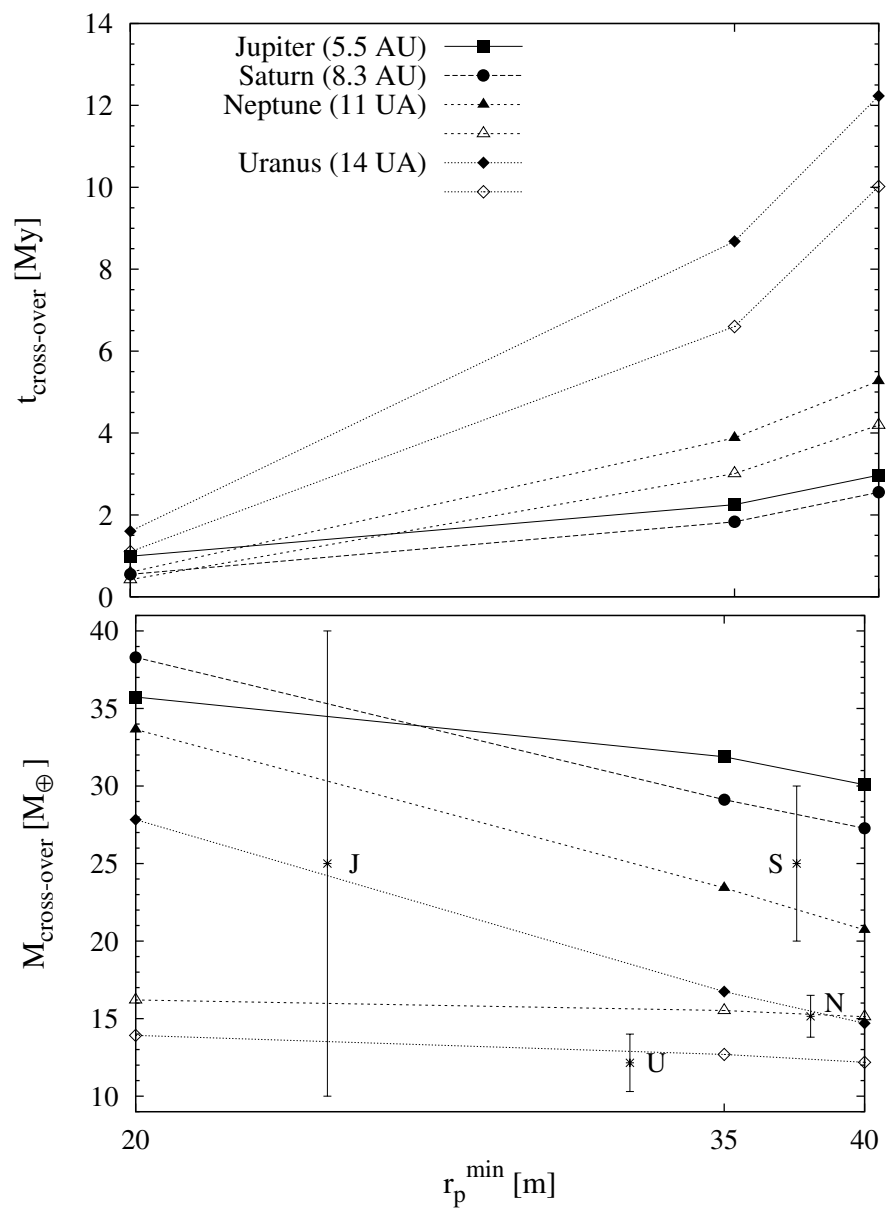

Fig. 8. Cross-over time (top) and cross-over mass (bottom) as function of the minimum radius of the size distribution of planetesimals for the simultaneous formation. Here the disk surface density is characterized by $\Sigma \propto a^{-3 / 2}$. For $r_{\mathrm{p}}^{\mathrm{min}}=35$ and $40 \mathrm{~m}$ the cross-over times and crossover masses of Jupiter and Saturn agree well with the observational and theoretical estimates. The cross-over masses of Neptune and Uranus are larger than their current total masses. However, for both planets the mass of the core at the time they reach their current masses (open triangles and diamonds) agree with theoretical estimates.

We remark that the formation time-scale of the gas giants was shorter than that of the ice giant.

\subsection{The case of $\Sigma \propto a^{-1}$}

In this section we repeat the previous method but now with a surface density profile for the disk of $\Sigma \propto a^{-1}$. The disk is then defined by

$$
\begin{aligned}
\Sigma_{\mathrm{s}}(a)= & \left\{15.125+(60.5-15.125)\left[\frac{1}{2} \tanh \left(\frac{a-2.7}{0.5}\right)+\frac{1}{2}\right]\right\} \\
& \times\left(\frac{a}{1 \mathrm{AU}}\right)^{-1} \mathrm{~g} \mathrm{~cm}^{-2} \\
\Sigma_{\mathrm{g}}(a)= & 3954.25\left(\frac{a}{1 \mathrm{AU}}\right)^{-1} \mathrm{~g} \mathrm{~cm}^{-2} .
\end{aligned}
$$

The initial mass of the disk is $\sim 0.082 M_{\odot}$.

In Table 5 and Fig. 9 we show the results corresponding to the isolated formation of each planet. Jupiter reached its crossover mass in less than 10 Myr for $10 \mathrm{~m} \lesssim r_{\mathrm{p}}^{\min } \lesssim 800 \mathrm{~m}$, Saturn for $10 \mathrm{~m} \lesssim r_{\mathrm{p}}^{\min } \lesssim 400 \mathrm{~m}$, while Neptune and Uranus 
Table 5. Isolated formation of the solar system giant planet cores for a disk with a surface density of solids and gas $\propto a^{-1}$.

\begin{tabular}{lccccccccc}
\hline \hline & Jupiter & \multicolumn{3}{c}{ Saturn } & \multicolumn{3}{c}{ Neptune } & \multicolumn{3}{c}{ Uranus } \\
$r_{\mathrm{p}}^{\text {min }}$ & $M_{\text {over }}^{\text {cross }}$ & $t_{\text {over }}^{\text {cross }}$ & $M_{\text {over }}^{\text {cross }}$ & $t_{\text {over }}^{\text {cross }}$ & $\begin{array}{c}M_{\text {over }}^{\text {coros }} \\
t_{\text {over }}^{\text {cross }}\end{array}$ & $M_{\text {over }}^{\text {cross }}$ & $t_{\text {over }}^{\text {cross }}$ \\
{$[m]$} & {$\left[M_{\oplus}\right]$} & {$[\mathrm{Myr}]$} & {$\left[M_{\oplus}\right]$} & {$[\mathrm{Myr}]$} & {$\left[M_{\oplus}\right]$} & {$[\mathrm{Myr}]$} & {$\left[M_{\oplus}\right]$} & {$[\mathrm{Myr}]$} \\
\hline 10 & 24.66 & 10.49 & 25.38 & 3.00 & 30.71 & 0.59 & 42.56 & 0.09 \\
& & & & & $(15.48)$ & $(0.20)$ & $(14.20)$ & $(0.05)$ \\
50 & 26.32 & 2.31 & 34.41 & 0.68 & 32.81 & 0.85 & 30.64 & 1.19 \\
& & & & & $(15.75)$ & $(0.63)$ & $(13.61)$ & $(0.90)$ \\
100 & 27.46 & 2.18 & 28.39 & 1.84 & 26.20 & 2.55 & 23.55 & 3.80 \\
& & & & & $(15.31)$ & $(2.19)$ & $(13.17)$ & $(3.21)$ \\
150 & 25.99 & 2.47 & 25.16 & 2.82 & 22.51 & 4.36 & 19.52 & 7.09 \\
& & & & & $(14.87)$ & $(3.85)$ & $(12.72)$ & $(6.16)$ \\
200 & 25.00 & 2.82 & 22.94 & 3.85 & 19.85 & 6.45 & 16.48 & 11.49 \\
& & & & & $(14.40)$ & $(5.81)$ & $(12.20)$ & $(10.25)$ \\
400 & 21.06 & 4.61 & 17.28 & 9.11 & - & - & - & - \\
800 & 16.16 & 9.78 & - & - & - & - & - & - \\
\hline
\end{tabular}

Table 6. Same as Table 5 but for the simultaneous formation of the giant planets.

\begin{tabular}{cccccccccc}
\hline \hline \multicolumn{3}{c}{ Jupiter } & \multicolumn{3}{c}{ Saturn } & \multicolumn{3}{c}{ Neptune } & \multicolumn{3}{c}{ Uranus } \\
$r_{\mathrm{p}}^{\text {min }}$ & $M_{\text {over }}^{\text {cross }}$ & $t_{\text {over }}^{\text {cross }}$ & $M_{\text {over }}^{\text {cross }}$ & $t_{\text {over }}^{\text {cross }}$ & $M_{\text {over }}^{\text {cooss }}$ & $t_{\text {over }}^{\text {cross }}$ & $M_{\text {over }}^{\text {cross }}$ & $t_{\text {over }}^{\text {cross }}$ \\
{$[m]$} & {$\left[M_{\oplus}\right]$} & {$[\mathrm{Myr}]$} & {$\left[M_{\oplus}\right]$} & {$[\mathrm{Myr}]$} & {$\left[M_{\oplus}\right]$} & {$[\mathrm{Myr}]$} & {$\left[M_{\oplus}\right]$} & {$[\mathrm{Myr}]$} \\
\hline 100 & 32.03 & 2.14 & 28.53 & 1.98 & 26.97 & 2.81 & 24.25 & 4.18 \\
& & & & & $(15.61)$ & $(2.27)$ & $(13.50)$ & $(3.13)$ \\
150 & 28.87 & 2.73 & 23.85 & 2.80 & 22.01 & 4.97 & 19.08 & 7.15 \\
& & & & & $(15.23)$ & $(4.11)$ & $(12.90)$ & $(5.90)$ \\
200 & 26.54 & 2.96 & 19.17 & 3.82 & 18.83 & 7.68 & 16.15 & 11.26 \\
& & & & & $(14.65)$ & $(6.43)$ & $(12.40)$ & $(9.55)$ \\
\hline
\end{tabular}

reached their cross-over mass in less than 10 Myr for $10 \mathrm{~m} \lesssim$ $r_{\mathrm{p}}^{\min } \lesssim 200 \mathrm{~m}$. Accordingly, the isolated formation for $r_{\mathrm{p}}^{\min }$ between $10 \mathrm{~m}$ and $200 \mathrm{~m}$ of the four planets occurred in less than 10 Myr.

According to these results we calculated the simultaneous formation of the giant planets for a size distribution of planetesimals between $r_{\mathrm{p}}^{\mathrm{min}}$ and $100 \mathrm{~km}$, where we adopted for $r_{\mathrm{p}}^{\min }$ several discrete values between $10 \mathrm{~m}$ and $200 \mathrm{~m}$.

The rapid formation of the outer planets significantly augmented the planetesimal accretion rate of Jupiter and Saturn for the size distributions of planetesimals between $10 \mathrm{~m}-100 \mathrm{~km}$ and $50 \mathrm{~m}-100 \mathrm{~km}$. The planetesimal accretion rate became so high $\left(10^{-3}-10^{-2} M_{\oplus} \mathrm{yr}^{-1}\right)$ that models corresponding to Jupiter and Saturn did not converge. We note that the masses of the cores corresponding to Jupiter and Saturn were larger before models failed to converge than the total heavy element corresponding to Jupiter $\left(\sim 40 M_{\oplus}\right)$ and Saturn $\left(\sim 30 M_{\oplus}\right)$, respectively.

On the other hand, for $r_{\mathrm{p}}^{\min }=100,150$ and $200 \mathrm{~m}$, the results for the simultaneous formation were similar to the isolated ones (see Table 6). We remark that for these cases our results for both the cross-over times and cross-over masses of Jupiter and Saturn were nicely agreed with the observations and theoretical estimates, respectively (Fig. 10). Again, the cross-over masses of Uranus and Neptune were larger than their current masses, but the core masses at the time they reached their current masses agreed with theoretical estimates. We note again the important fact that the formation time-scale of the gas giants was shorter than that of the ice giants.

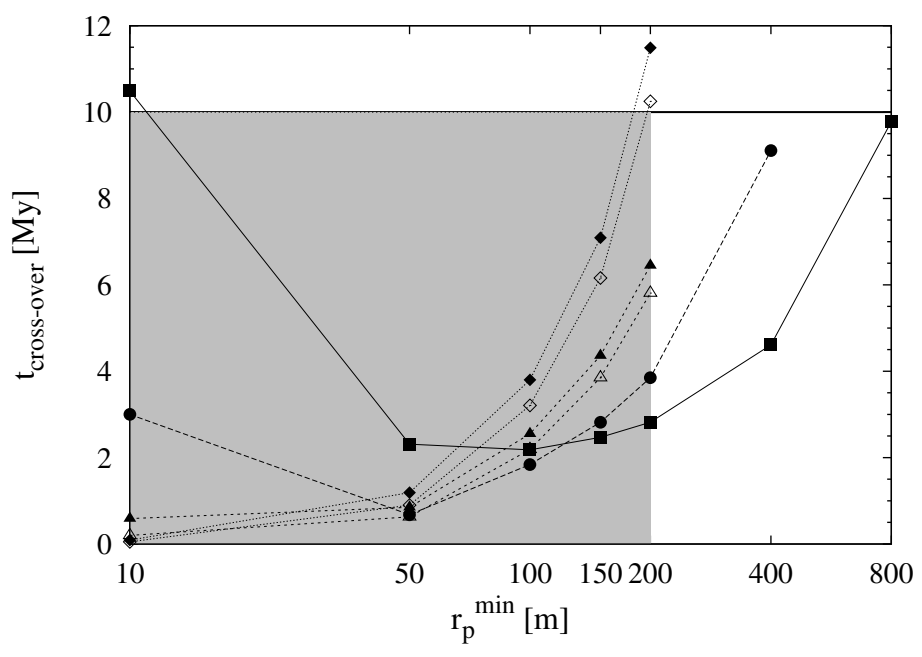

Fig. 9. Same as Fig. 5 but for a disk with a surface density profile $\Sigma \propto a^{-1}$.
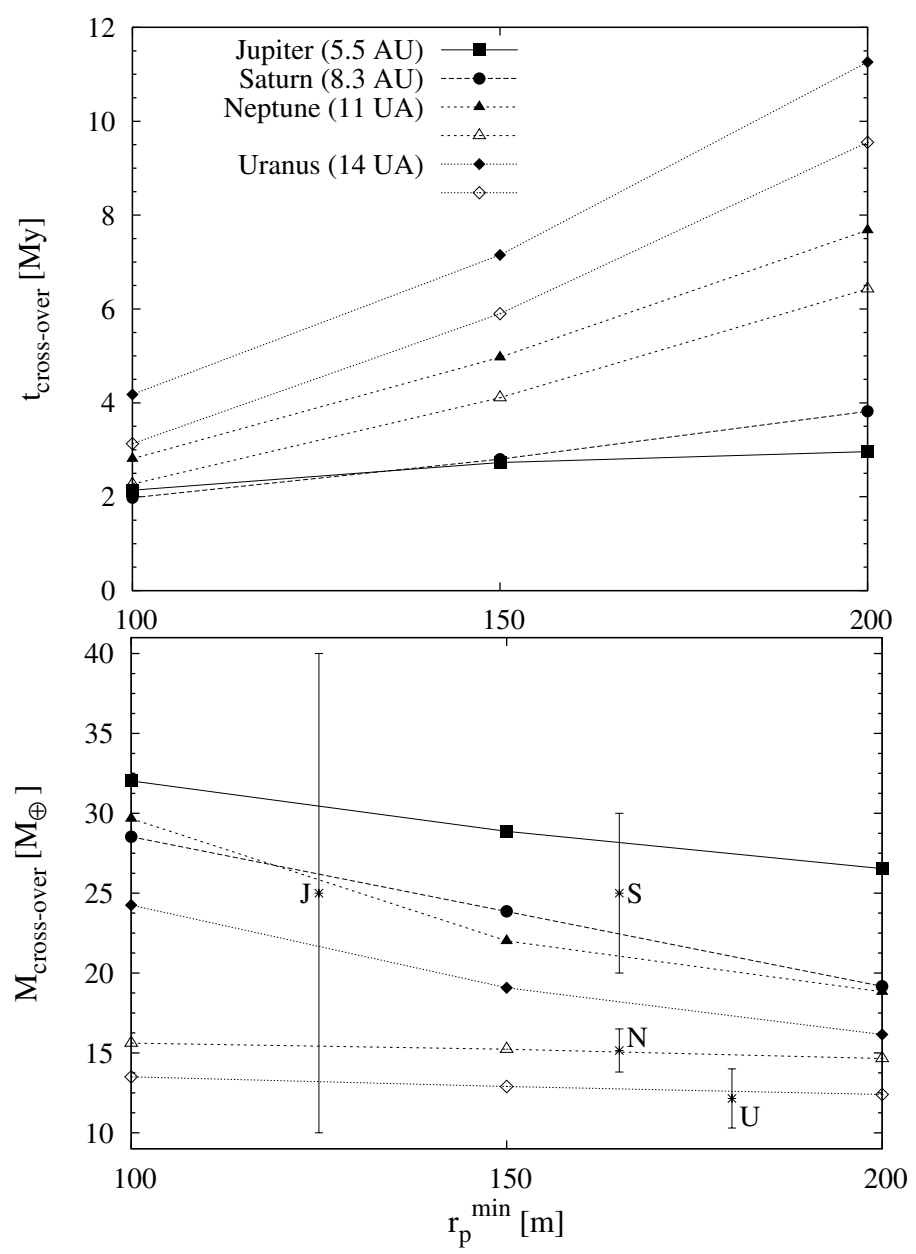

Fig. 10. Cross-over time (top) and cross-over mass (bottom) as function of the minimum radius of the size distribution of planetesimals for the simultaneous formation. Here the disk surface density is characterized by $\Sigma \propto a^{-1}$. For all cases the cross-over times and cross-over masses of Jupiter and Saturn agree well with the observational and theoretical estimates. The cross-over masses of Neptune and Uranus are higher than their current total masses. However, for both planets the mass of the core at the time they reach their current masses (open triangles and diamonds) agree with theoretical estimates. 
Table 7. Isolated formation of the giant planets for a disk with a surface density of solids and gas $\propto a^{-1 / 2}$.

\begin{tabular}{|c|c|c|c|c|c|c|c|c|}
\hline \multirow{3}{*}{$\begin{array}{l}r_{\mathrm{p}}^{\min } \\
{[m]}\end{array}$} & \multicolumn{2}{|l|}{ Jupiter } & \multicolumn{2}{|l|}{ Saturn } & \multicolumn{2}{|l|}{ Neptune } & \multicolumn{2}{|l|}{ Uranus } \\
\hline & $M_{\text {over }}^{\text {cross }}$ & $t_{\text {over }}^{\text {corss }}$ & $M_{\text {over }}^{\text {cross }}$ & $t_{\mathrm{over}}^{\text {cross }}$ & $M_{\mathrm{over}}^{\text {cross }}$ & $t_{\text {over }}^{\text {cross }}$ & $M_{\text {over }}^{\text {cross }}$ & $t_{\mathrm{over}}^{\text {cross }}$ \\
\hline & {$\left[M_{\oplus}\right]$} & [Myr] & {$\left[M_{\oplus}\right]$} & [Myr] & {$\left[M_{\oplus}\right]$} & [Myr] & {$\left[M_{\oplus}\right]$} & {$[\mathrm{Myr}]$} \\
\hline \multirow[t]{2}{*}{10} & 25.27 & 7.62 & 26.00 & 2.33 & 34.55 & 0.09 & 59.85 & 0.03 \\
\hline & & & & & (16.50) & $(0.03)$ & $(14.30)$ & $(0.02)$ \\
\hline \multirow[t]{2}{*}{50} & 28.65 & 2.14 & 42.00 & 0.10 & 54.15 & 0.06 & 55.50 & 0.06 \\
\hline & & & & & (16.52) & $(0.04)$ & $(14.20)$ & $(0.04)$ \\
\hline \multirow[t]{2}{*}{100} & 31.31 & 2.02 & 35.00 & 0.77 & 36.73 & 0.44 & 38.21 & 0.30 \\
\hline & & & & & (15.99) & $(0.30)$ & (13.95) & $(0.17)$ \\
\hline \multirow[t]{2}{*}{500} & 23.86 & 4.19 & 23.65 & 4.42 & 23.32 & 4.53 & 23.38 & 4.00 \\
\hline & & & & & (14.95) & (4.03) & (13.14) & (3.37) \\
\hline \multirow[t]{2}{*}{1000} & 18.64 & 8.73 & 17.88 & 10.14 & 17.57 & 10.65 & 17.83 & 9.72 \\
\hline & & & & & (13.81) & $(9.86)$ & (12.42) & $(8.60)$ \\
\hline
\end{tabular}

Table 8. Same as Table 7 but for the simultaneous formation.

\begin{tabular}{ccccccccc}
\hline \hline & Jupiter & \multicolumn{3}{c}{ Saturn } & \multicolumn{3}{c}{ Neptune } & \multicolumn{3}{c}{ Uranus } \\
$r_{\mathrm{p}}^{\text {min }}$ & $M_{\text {over }}^{\text {cross }}$ & $t_{\text {over }}^{\text {cross }}$ & $M_{\text {over }}^{\text {cross }}$ & $t_{\text {over }}^{\text {cross }}$ & $M_{\text {over }}^{\text {cross }}$ & $t_{\text {over }}^{\text {cross }}$ & $M_{\text {over }}^{\text {cross }}$ & $t_{\text {over }}^{\text {cross }}$ \\
{$[m]$} & {$\left[M_{\oplus}\right]$} & {$[\mathrm{Myr}]$} & {$\left[M_{\oplus}\right]$} & {$[\mathrm{Myr}]$} & {$\left[M_{\oplus}\right]$} & {$[\mathrm{Myr}]$} & {$\left[M_{\oplus}\right]$} & {$[\mathrm{Myr}]$} \\
\hline 500 & 53.37 & 2.42 & 35.54 & 2.37 & 22.62 & 2.92 & 23.20 & 4.50 \\
& & & & & $(15.67)$ & $(2.29)$ & $(13.04)$ & $(3.78)$ \\
1000 & 47.90 & 4.80 & 31.05 & 4.73 & 16.85 & 6.07 & 16.21 & 11.50 \\
& & & & & $(13.87)$ & $(5.06)$ & $(12.24)$ & $(10.09)$ \\
\hline
\end{tabular}

\subsection{The case of $\Sigma \propto a^{-1 / 2}$}

Finally, we repeated the calculations employing a very flat surface density profile, proportional to $a^{-1 / 2}$. In this case, the resulting expressions for $\Sigma$ are

$$
\begin{aligned}
\Sigma_{\mathrm{s}}(a)= & \left\{6.45+(25.8-6.45)\left[\frac{1}{2} \tanh \left(\frac{a-2.7}{0.5}\right)+\frac{1}{2}\right]\right\} \\
& \times\left(\frac{a}{1 \mathrm{AU}}\right)^{-1 / 2} \mathrm{~g} \mathrm{~cm}^{-2} \\
\Sigma_{\mathrm{g}}(a)= & 1686.25\left(\frac{a}{1 \mathrm{AU}}\right)^{-1 / 2} \mathrm{~g} \mathrm{~cm}^{-2}
\end{aligned}
$$

The initial mass of the disk in this case was $\sim 0.13 M_{\odot}$.

In Table 7 we show the results for the isolated formation of each planet. We see that each planet reached their cross-over mass in less than $\sim 10 \mathrm{Myr}$ with a size distribution of planetesimals ranging from $r_{\mathrm{p}}^{\min }$ to $100 \mathrm{~km}$, where $r_{\mathrm{p}}^{\min }$ could take values between $10 \mathrm{~m}$ and $1 \mathrm{~km}$ (Fig. 11). However, there were cases for this density profile where the final solid masses in the planets' interior were higher than the theoretical estimates.

The results of the simultaneous formation for this profile are summarized in Table 8. For $r_{\mathrm{p}}^{\mathrm{min}}$ between 10 and $100 \mathrm{~m}$, the fast formation of Neptune and Uranus significantly augmented the planetesimal accretion rate of Jupiter and Saturn. This rate became so high $\left(10^{-3}-10^{-2} M_{\oplus} \mathrm{yr}^{-1}\right)$ that models corresponding to Jupiter and Saturn failed to converge. We note again that the mass of the cores corresponding to Jupiter and Saturn before models failed to converge were larger than the total heavy element corresponding to Jupiter $\left(\sim 40 M_{\oplus}\right)$ and Saturn $\left(\sim 30 M_{\oplus}\right)$, respectively. For $r_{\mathrm{p}}^{\mathrm{min}}=500 \mathrm{~m}$ and $r_{\mathrm{p}}^{\min }=1 \mathrm{~km}$ the presence of Uranus reduced the formation time of the inner planets by a factor of 2, but significantly increased its final solid masses. While the cross-over time of Jupiter and Saturn nicely agree with theoretical estimates, the cross-over masses corresponding to Jupiter and Saturn are larger than the total heavy element content corresponding to Jupiter $\left(\sim 40 M_{\oplus}\right)$ and Saturn $\left(\sim 30 M_{\oplus}\right)$.

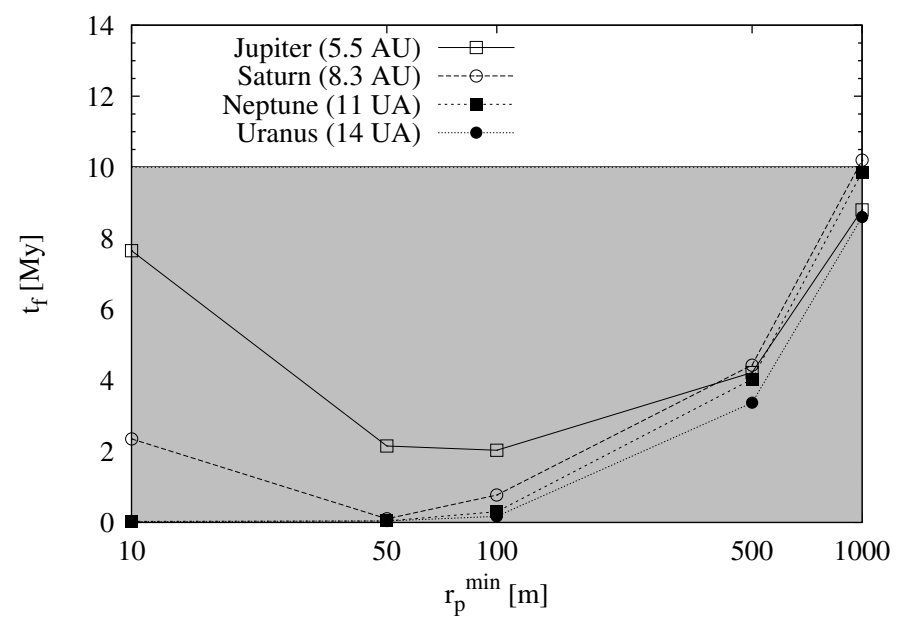

Fig. 11. Same as Figs. 5 and 9 but for a disk with a surface density profile $\Sigma \propto a^{-1 / 2}$.

\section{Discussion and conclusions}

We studied the in situ simultaneous formation of the solar system giant planets in the framework of the core accretion model and according to the conditions imposed by the Nice model. The goal of this work was to delimit several parameters that describe the protoplanetary disk (especially the surface density profile and planetesimal's population size distribution) in relation to the likelihood of the formation of the four giant planet's cores, in less than $\sim 10$ Myr and with a content of heavy elements in good agreement with current estimatess.

The surface density profile of the disk was assumed as a power law, $\Sigma \propto a^{-p}$, where the power index $p$ is considered as a free parameter, and several values of $p$ were analyzed. We considered that accreted planetesimals follow a power law mass distribution of the form $n(m) \propto m^{-2.5}$, where most of the mass is in smaller objects. We assumed the planetesimals to be spheres of constant density, which in turn implies a power law distribution for the planetesimals' radii. We discretized the continuous planetesimal population, considering that the minimum radius, $r_{\mathrm{p}}^{\min }$, is a free parameter as well and the maximum radius is fixed at $r_{\mathrm{p}}^{\max }=100 \mathrm{~km}$.

We note that, although we calculated the evolution of the planetesimal disk including the planet's accretion and planetesimal migration, we excluded aggregation and fragmentation. Inaba et al. (2003) found that large amounts of mass could be lost by the inward drift of small collision fragments. This means that fragmentation seems to play an important role and should be considered in a more accurate model. Although we cannot predict how fragmentation could affect the results of our model, we point out some differences between the working hypothesis of Inaba et al. (2003) and the one presented here. Inaba et al. (2003) started with an homogeneous population of planetesimals of radii $\sim 10 \mathrm{~km}$. Here, smaller planetesimals appear because of fragmentation of bigger ones. This implies that most of the mass resides in bigger planetesimals (Dohnanyi 1969; Wetherill $\&$ Stewart 1993). On the other hand, we started our simulations already in the oligarchic growth regime, with an embryo with the mass of the Moon $\left(\sim 0.01 M_{\oplus}\right)$ immersed in a swarm of planetesimals that follow a mass distribution. Ormel et al. (2010), employing statistical simulations that include several physical processes such as dynamical friction, viscous stirring, gas drag, and fragmentation, found that starting with an homogeneous populations of planetesimals of radii $r_{0}$, the transition between 
the runaway growth and oligarchic growth is characterized by a power-law size distribution of mass index $q \sim 2.5$, where most of the mass lies in small planetesimals. Consequently, our initial conditions are consistent with the oligarchic growth regime using $r_{0} \lesssim 1 \mathrm{~km}$. Another important difference is the moment at which the gas drag of the envelope becomes efficient. Inaba et al. (2003) found that when the mass of the embryo approximately reaches the mass of Mars, it is capable to acquire an envelope. However, we found that when the mass of the embryos is $\sim 0.1 M_{\oplus}$, it already has an envelope and the envelope gas drag becomes efficient for smaller planetesimals (several tens and hundreds of meters sized planetesimals). Moreover, as we can see in Fig. 12, when the mass of the embryo is $\sim 0.6 M_{\oplus}$, the ratio between the enhanced radius and the core radius is a factor $\sim 7$. The enhancement of the cross-section capture owing to the presence of the envelope and the moment when it becomes efficient are very important. As an example, we calculated the isolated formation of Jupiter for a disk with density profiles $\propto a^{-1}$, and with a planetesimal size distribution between $100 \mathrm{~m}$ and $100 \mathrm{~km}$, but now not taking into account the enhancement of the capture cross-section owing to the envelope gas drag. We compared this simulation to the one with the same initial conditions but considering the enhancement of the capture cross-section. In Fig. 13 we show the comparison between the two simulations. If the enhanced capture cross-section was considered, Jupiter reached its cross-over mass at 2.18 Myr (the value of the cross-over mass is $27.46 M_{\oplus}$, see Table 5), while if the enhanced capture cross-section was excluded, Jupiter did not reach its cross-over mass (the simulation was halted at $15 \mathrm{Myr}$ and Jupiter only reached a core of $\sim 2.5 M_{\oplus}$ with a negligible envelope). Evidently, when the core reached the mass of Mars (for the case where the enhancement of the capture cross-section is not incorporated) the corresponding core for the case where the enhanced capture cross-section is considered is able to grow up to $10 M_{\oplus}$ in the same elapsed time. Clearly the enhancement of the cross-section of the core owing to the presence of the envelope plays a fundamental role in the formation of a giant planet. Moreover, the planetesimal accretion rate suffers a significant drop when the enhancement of the cross-section is not included. Finally, the difference between both simulations begins when the envelope gas drag becomes effective for small planetesimals (at $\sim 0.01 \mathrm{Myr}$, which corresponds to a core mass of $\sim 0.1 M_{\oplus}$ ). Consequently, the moment (or the mass of the embryo) at which the envelope gas drag becomes effective for smaller planetesimals is important because we are considering that most of the mass lies in small objects. The difference in the ratio between the enhanced radius and the core radius with respect to Inaba et al. (2003) may arise because we are employing a more accurate model to calculate the growth and evolution of the planet's envelope. Therefore, we conclude that fragmentation could be an important effect, and we plan to include it in future improvements of our model, but we think that a definitive evaluation of its relevance should await calculations including a realistic model of the process, combined with a detailed prescription of the effect of the planet atmosphere on the fragments.

We started studying a Desch-like profile for the disk surface density, $\Sigma \propto a^{-2}$ (see Desch 2007). We first computed the isolated formation of the four giant planets looking for a common interval for the minimum radius of the planetesimal population that could allow the simultaneous formation of all the planets. However, we did not find overlapping values for the four planets. Despite that, we calculated some simulations to check if the simultaneous formation was possible (see Table 2), and confirmed that according to our model, adopting Desch profile makes the

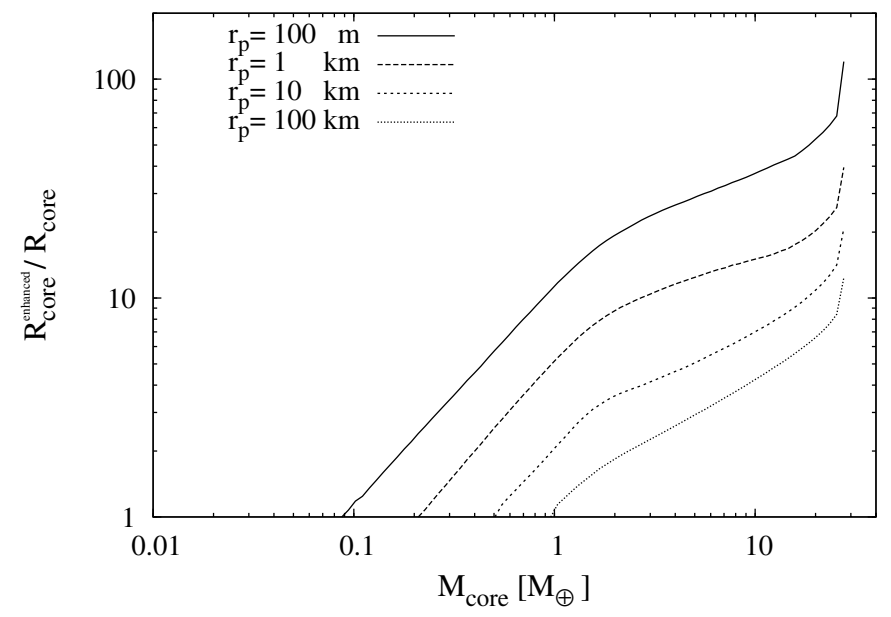

Fig. 12. Ratio between the enhanced radius and the core radius as function of the core mass for the isolated formation of Jupiter, for a disk with a density profile $\propto a^{-1}$, and with a planetesimal size distribution between $100 \mathrm{~m}$ and $100 \mathrm{~km}$. The envelope gas drag for smaller planetesimals becomes effective at very small core masses.

formation of the giant planets very unlikely, because the formation time-scale largely exceeds the $10 \mathrm{Myr}$ barrier.

We proceeded then with less steep profiles. We analyzed another three cases: $\Sigma \propto a^{-1.5}, \Sigma \propto a^{-1}$ and $\Sigma \propto a^{-0.5}$, the first one corresponding to the standard minimum mass solar nebula and the other two following results of Andrews et al. (2009, 2010), which are based on observational data of circumstellar disks. In all these cases, when first calculating the isolated formation of Jupiter, Saturn, Neptune and Uranus, we were able to find several common values for $r_{\mathrm{p}}^{\mathrm{min}}$ that could lead to a satisfactory result when calculating the simultaneous formation (see Figs. 5, 9, 11). Indeed, for these values we ran the corresponding simulations for the simultaneous formation. For these three density profiles, the simultaneous formation could be, in principle, considered as possible if the most abundant planetesimals are those whose radii range from several tens to several hundreds of meters depending on the case, but not larger than that. From our results it is clear that the shallower the profile density of the disk, the more efficient the formation process. Moreover, considering lower values for $p$ allowed us to increase the minimum radius of the accreted planetesimals. We note that decreasing $p$ is directly related to considering more massive nebulae, which in turn means that the mass in the protoplanets feeding zone is larger, favoring the accretion process in this way. Furthermore, planetesimal migration is less efficient because the gas drag is weaker, so planetesimals remain longer in the feeding zone of the protoplanets. Besides this, for a solid surface density profile $\Sigma_{\mathrm{s}} \propto a^{-p}$ where $p<1$, the mass of solids grows outward from the planetesimal disk, and the incoming mass flux in the feeding zone of the protoplanets exceeds the outgoing one, which also favors the accretion.

On the other hand, Desch's profile is compatible with a decretion disk. This type of profiles turned out to be almost stationary for about ten million years. Crida (2009) demonstrated that in a disk with this density profile, the four giant planets of the solar system would have been unable to survive. Particularly, Jupiter would have become a hot giant planet. Morbidelli \& Crida (2007) and Crida (2009) also showed that Jupiter and Saturn could avoid migration if a less massive nebula, such as Hayashi's nebula is assumed. While the initial value of the gas density used in our simulations is about five times higher than 

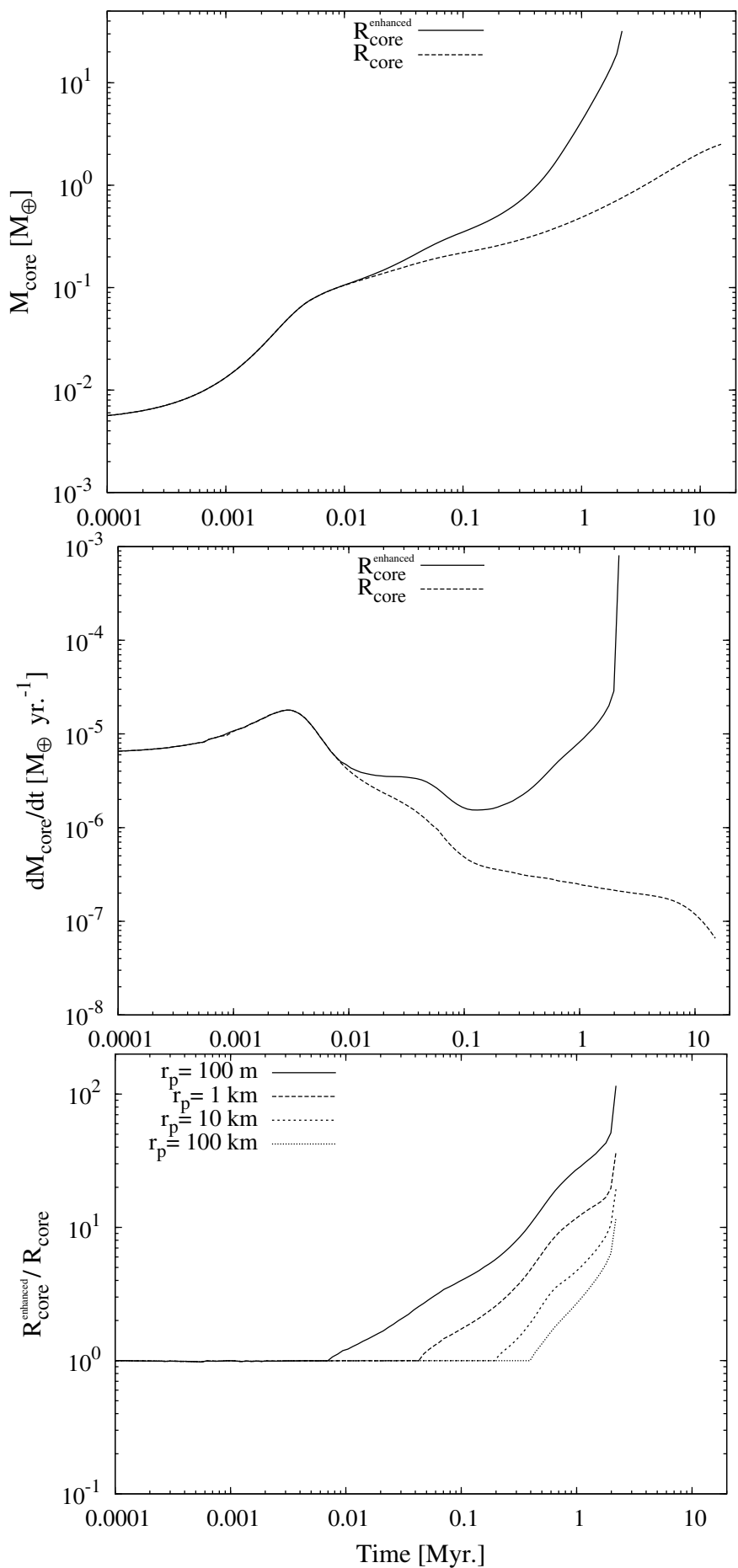

Fig. 13. Top and middle: time evolution of the core mass and the planetesimal accretion rate for the isolated formation of Jupiter employing a disk with density profiles $\propto a^{-1}$, and a planetesimal size distribution between $100 \mathrm{~m}$ and $100 \mathrm{~km}$, with (solid line) and without (dashed line) considering the enhancement of the capture cross-section owing to the envelope gas drag. Bottom: the ratio between the enhanced radius and the core radius as function of time for the case where the enhancement of the capture cross-section is incorporated.

that of Hayashi's minimum mass solar nebula at the position of Jupiter, this value is decreased by an exponential dissipation factor during the formation of the planets. Then, by the time Jupiter and Saturn finished their formation, the disk conditions correspond to a much less massive disk, especially in the cases where the formation times of Jupiter and Saturn are similar to the dis- sipation time-scale of the gas disk. This should help to prevent Jupiter and Saturn migration in initially massive protoplanetary disks.

We found several values of $r_{\mathrm{p}}^{\min }$ for each profile considered here (except $p=2$ ) for which the simultaneous formation of the planets could be achieved in less than $10 \mathrm{Myr}$ and the amount of heavy elements in the interior of the planets agreed well with theoretical estimates of current abundances. In most of the cases, the formation time-scale of the gas giants was shorter than the formation time-scale of the ice giants. However, when very small planetesimals were the most abundant bodies in the disk, the icy giants formed before the gas giants. We consider these cases very unlikely, at least under the hypothesis of our model, because they would need extra explanations to justify why they did not start the gaseous runaway before Jupiter and Saturn if, in principle, there was still plenty of material that could be accreted. This specially applies for the cases where Uranus and/or Neptune were the first to be formed.

There is another interesting result we would like to highlight. When we consider $\Sigma \propto a^{-1}$ and $r_{\mathrm{p}}^{\mathrm{min}}=100 \mathrm{~m}$ (Sect. 4.2) the cross-over times of Jupiter and Saturn and the time at which Neptune and Uranus reached their current masses are of the same time-scale; around 2-3 Myr (see Table 6 and Fig. 10). This time-scale is shorter than the dissipation time-scale of the disk. That the four planets formed on the same time-scale, which is also comfortably short, is not a minor point, but on the contrary presents a scenario that should be explored in more detail in the future. If the gaseous component of the disk could be rapidly evaporated by some external mechanism (for instance, if the solar system is strongly irradiated by an OB stellar association (see, for example, the work by Clarke 2007)), our results can provide a pathway to find suitable parameters to describe the protoplanetary disk. Therefore, we note that there are cases where calculations of simultaneous formation with an appropriate planetesimal size distribution, together with a flat surface density profile provide suitable conditions for the timely formation of the external planets, the four formation processes occurring on the same time-scale.

Finally, we would like to remark that it is necessary that the size distribution of planetesimals extends to objects with radii $<1 \mathrm{~km}$ to form the four planets on a time-scale compatible with the observational estimates. Goldreich et al. (2004) already pointed out that the presence of a large amount of small planetesimals would help to accelerate planetary formation. If the planetesimal disk is dominated by big bodies, the time-scale to form solid embryos able to bind a significant envelope and start the gaseous runaway accretion would be too long to complete the formation in less than $10 \mathrm{Myr}$.

However, this result contradicts recent models for planetesimal formation. Johansen et al. (2007), Cuzzi et al. (2008) and Youdin (2011) describe different mechanisms for planetesimal formation that incorporate turbulence as a way to aid planetesimals to grow, showing that if planetesimals formed this way, they are likely to be large $(\sim 100 \mathrm{~km}$, or even bigger, growing directly from sub-m particles). However, these models do not predict a specific planetesimal size distribution and the nature of the turbulence is controversial. Nelson \& Gressel (2010) showed that fully developed magneto-hydrodynamic turbulence in protoplanetary disks would have a destructive effect on embedded planetesimals. Arguments in favor of the hypothesis of primitive initial large planetesimals are found in Morbidelli et al. (2009b), where through models of the collisional evolution of the asteroid belt the authors conclude that the primitive asteroids should have been big. However, their model does not 
take a very important effect into account: the primitive intense bombardment of the asteroid belt by outer planetary region comets (Gil et al. 1999), which should change this conclusion. Moreover, Weidenschilling (2011) proposed an alternative scenario to the work of Morbidelli et al. (2009b). Weidenschilling found that coagulation from small planetesimals $(\sim 100 \mathrm{~m}$ of diameter) better represents the size distribution of the asteroid belt. These small planetesimals could be formed by coagulation in collision-driven processes by size-dependent drift caused by nebular gas drag (Weidenschilling 1997). For these reasons, we consider that the planetesimal size problem is far from being fully understood, and a distribution where most of the solid mass accreted by a protoplanet comes from small planetesimals cannot be ruled out.

However, one possible way to relax this assumption is to consider giant impacts and mergers during the planetary formation (Li et al. 2010). If a forming giant planet is impacted by a Mars- to an Earth-mass embryo, the impactor most likely reaches the core, possibly remixing the core into the envelope (Li et al. 2010). Broeg \& Benz (2011) have studied the effect of this impact on the gas accretion rate: initially, most of the envelope can be ejected, but afterward gas is reaccreted very fast and the overall gas accretion rate turns out to be larger than in the standard, continuous-planetesimal-accretion scenario. As a consequence, the planet gains large amounts of mass in a short time-scale and the growth-time-scale is reduced.

Another path to accelerate the formation of the gaseous planets is to consider the fusion of embryos as a mechanism to obtain massive cores, especially in the early stages of the disk evolution. Oligarchic growth predicts the simultaneous formation of many embryos on orbits separated by about 10 Hill radii from each other. Several works have been done to study the fusion of growing protoplanets, but only taking into account their solid cores, neglecting the presence of the envelope (Chambers 2006; Brunini \& Benvenuto 2008). To perform a full-detailed simulation in the context of the present study we would need to compute the merger of embryos self-consistently, taking into account the growing gaseous envelope. This is a very complex phenomenon, beyond the scope of the present paper, but we will investigate this in a future work.

Acknowledgements. We wish to acknowledge the useful comments of C. Broeg, A. Crida, L. Fouchet, Y. Alibert, and Y. Miguel. We also appreciate the constructive criticism that John Chambers (the referee) made about our work, which had helped us to significantly improve this work. A.F. was supported by the European Research Council under grant number 239605.

\section{References}

Adachi, I., Hayashi, C., \& Nakazawa, K. 1976, Progress of Theoretical Physics, 56,1756

Alibert, Y., Mousis, O., Mordasini, C., \& Benz, W. 2005, ApJ, 626, L57

Andrews, S. M., Wilner, D. J., Hughes, A. M., Qi, C., \& Dullemond, C. P. 2009 , ApJ, 700, 1502

Andrews, S. M., Wilner, D. J., Hughes, A. M., Qi, C., \& Dullemond, C. P. 2010, ApJ, 723, 1241

Batygin, K., \& Brown, M. E. 2010, ApJ, 716, 1323
Benvenuto, O. G., \& Brunini, A. 2005, MNRAS, 356, 1383

Benvenuto, O. G., Fortier, A., \& Brunini, A. 2009, Icarus, 204, 752

Broeg, C., \& Benz, W. 2011, A\&A, submitted

Brunini, A., \& Benvenuto, O. G. 2008, Icarus, 194, 800

Chambers, J. 2006, Icarus, 180, 496

Chambers, J. E. 2010, Icarus, 208, 505

Clarke, C. J. 2007, MNRAS, 376, 1350

Crida, A. 2009, ApJ, 698, 606

Cuzzi, J. N., Hogan, R. C., \& Shariff, K. 2008, ApJ, 687, 1432

Desch, S. J. 2007, ApJ, 671, 878

Dohnanyi, J. S. 1969, J. Geophys. Res., 74, 2531

Fortier, A., Benvenuto, O. G., \& Brunini, A. 2007, A\&A, 473, 311

Fortier, A., Benvenuto, O. G., \& Brunini, A. 2009, A\&A, 500, 1249

Gil-Hutton, R., \& Brunini, A. 1999, Planet. Space Sci., 47, 331

Goldreich, P., Lithwick, Y., \& Sari, R. 2004, ARA\&A, 42, 549

Gomes, R., Levison, H. F., Tsiganis, K., \& Morbidelli, A. 2005, Nature, 435, 466

Guilera, O. M., Brunini, A., \& Benvenuto, O. G. 2010, A\&A, 521, A50

Guillot, T. 2005, Ann. Rev. Earth Plan. Sci., 33, 493

Guillot, T., \& Gautier, D. 2009, Treatise of Geophysics, Planets and Moons, ed. G. Schubert, T. Spohn, 10, 439

Haisch, K. E., Jr., Lada, E. A., \& Lada, C. J. 2001, ApJ, 553, L153

Hayashi, C. 1981, Progress of Theoretical Physics Supplement, 70, 35

Inaba, S., Tanaka, H., Nakazawa, K., Wetherill, G. W., \& Kokubo, E. 2001, Icarus, 149, 235

Inaba, S., \& Ikoma, M. 2003, A\&A, 410, 711

Inaba, S., Wetherill, G. W., \& Ikoma, M. 2003, Icarus, 166, 46

Kley, W., Bitsch, B., \& Klahr, H. 2009, A\&A, 506, 971

Kokubo, E., \& Ida, S. 2000, Icarus, 143, 15

Johansen, A., Oishi, J. S., Mac Low, M.-M., et al. 2007, Nature, 448, 1022

Levison, H. F., Morbidelli, A., Vanlaerhoven, C., Gomes, R., \& Tsiganis, K. 2008, Icarus, 196, 258

Li, S. L., Agnor, C. B., \& Lin, D. N. C. 2010, ApJ, 720, 1161

Lodders, K., Palme, H., \& Gail, H. 2009, Landolt-Bornstein, New Series, A\&A, (Berlin: Springer Verlag) print version [arXiv: 0901.1149]

Machida, M. N., Kokubo, E., Inutsuka, S.-I., \& Matsumoto, T. 2010, MNRAS, 405,1227

Masset, F., \& Snellgrove, M. 2001, MNRAS, 320, L55

Miguel, Y., Guilera, O. M., \& Brunini, A. 2011, MNRAS, accepted [arXiv: 1106.3281]

Miguel, Y., Guilera, O. M., \& Brunini, A. 2011, MNRAS, 412, 2113

Militzer, B., Hubbard, W. B., Vorberger, J., Tamblyn, I., \& Bonev, S. A. 2008, ApJ, 688, L45

Morbidelli, A., \& Crida, A. 2007, Icarus, 191, 158

Morbidelli, A., Levison, H. F., Tsiganis, K., \& Gomes, R. 2005, Nature, 435, 462

Morbidelli, A., Tsiganis, K., Crida, A., Levison, H. F., \& Gomes, R. 2007, AJ, 134,1790

Morbidelli, A., Brasser, R., Tsiganis, K., Gomes, R., \& Levison, H. F. 2009a, A\&A, 507, 1041

Morbidelli, A., Bottke, W. F., Nesvorný, D., \& Levison, H. F. 2009b, Icarus, 204, 558

Nelson, R. P., \& Gressel, O. 2010, MNRAS, 409, 6398

Ohtsuki, K., Stewart, G. R., \& Ida, S. 2002, Icarus, 155, 436

Ormel, C. W., Dullemond, C. P., \& Spaans, M. 2010, ApJ, 714, L103

Paardekooper, S.-J., Baruteau, C., Crida, A., \& Kley, W. 2010, MNRAS, 401, 1950

Podolak, M., Podolak, J. I., \& Marley, M. S. 2000, Planet. Space Sci., 48, 143

Pollack, J. B., Hubickyj, O., Bodenheimer, P., et al. 1996, Icarus, 124, 62

Tanaka, H., Takeuchi, T., \& Ward, W. R. 2002, ApJ, 565, 1257

Thommes, E. W., Duncan, M. J., \& Levison, H. F. 2003, Icarus, 161, 431

Thommes, E. W., Matsumura, S., \& Rasio, F. A. 2008, Science, 321, 814

Tsiganis, K., Gomes, R., Morbidelli, A., \& Levison, H. F. 2005, Nature, 435, 459

Weidenschilling, S. J. 1977, Ap\&SS, 51, 153

Weidenschilling, S. J. 1997, Icarus, 127, 290

Weidenschilling, S. J. 2010, Lunar and Planetary Institute Science Conference Abstracts, 41, 1453

Wetherill, G. W., \& Stewart, G. R. 1993, Icarus, 106, 190

Youdin, A. N. 2011, ApJ, 731, 99 